\title{
TWO-DIMENSIONAL CURVATURE FUNCTIONALS WITH SUPERQUADRATIC GROWTH
}

\author{
ERNST KUWERT, TOBIAS LAMM, AND YUXIANG LI
}

\begin{abstract}
For two-dimensional, immersed closed surfaces $f: \Sigma \rightarrow \mathbb{R}^{n}$, we study the curvature functionals $\mathcal{E}^{p}(f)$ and $\mathcal{W}^{p}(f)$ with integrands $\left(1+|A|^{2}\right)^{p / 2}$ and $\left(1+|H|^{2}\right)^{p / 2}$, respectively. Here $A$ is the second fundamental form, $H$ is the mean curvature and we assume $p>2$. Our main result asserts that $W^{2, p}$ critical points are smooth in both cases. We also prove a compactness theorem for $\mathcal{W}^{p}$-bounded sequences. In the case of $\mathcal{E}^{p}$ this is just Langer's theorem [14, while for $\mathcal{W}^{p}$ we have to impose a bound for the Willmore energy strictly below $8 \pi$ as an additional condition. Finally, we establish versions of the Palais-Smale condition for both functionals.
\end{abstract}

\section{INTRODUCTION}

Let $\Sigma$ be a two-dimensional, closed differentiable manifold and $p>2$, hence $W^{2, p}\left(\Sigma, \mathbb{R}^{n}\right) \subset C^{1,1-\frac{2}{p}}\left(\Sigma, \mathbb{R}^{n}\right)$ by the Sobolev embedding theorem. On the open subset of immersions $W_{\mathrm{im}}^{2, p}\left(\Sigma, \mathbb{R}^{n}\right)$ we consider the two functionals

$$
\begin{aligned}
\mathcal{E}^{p}(f) & =\frac{1}{4} \int_{\Sigma}\left(1+|A|^{2}\right)^{\frac{p}{2}} d \mu_{g}, \\
\mathcal{W}^{p}(f) & =\frac{1}{4} \int_{\Sigma}\left(1+|H|^{2}\right)^{\frac{p}{2}} d \mu_{g} .
\end{aligned}
$$

Here $g$ denotes the first fundamental form with induced measure $\mu_{g}, A=\left(D^{2} f\right)^{\perp}$ the second fundamental form, and $H$ is the mean curvature vector. We prove regularity of critical points for both functionals.

Theorem 1.1. Let $f \in W_{\mathrm{im}}^{2, p}\left(\Sigma, \mathbb{R}^{n}\right)$ be a critical point of $\mathcal{W}^{p}$ or $\mathcal{E}^{p}$, where $2<$ $p<\infty$. Then local graph representations of $f$ are smooth, in fact real analytic.

In a graph representation, the Euler-Lagrange equations become fourth order elliptic systems, where the principal term has a double divergence structure. The systems are degenerate, in the sense that in both cases the coefficient of the principal term involves a $(p-2)$-th power of the curvature, which a priori may not be bounded. For the functional $\mathcal{W}^{p}(f)$, our first step towards regularity is an improvement of the integrability of $H$. For this we employ an iteration based on a new test function argument. More precisely, we solve the equation $L_{g} \varphi=|H|^{\lambda-1} H$ for appropriate $\lambda>1$ and then insert $\varphi$ as a test function. Here the operator $L_{g}=\sqrt{\operatorname{det} g} g^{\alpha \beta} \partial_{\alpha \beta}^{2}$ comes up in the principal term of the equation.

Date: November 6, 2018.

This research was supported in parts by the DFG Collaborative Research Center SFB/Transregio 71, and by a PIMS Postdoctoral Fellowship of the second author. 
Unfortunately, the same strategy does not apply in the case of the functional $\mathcal{E}^{p}(f)$, since then the corresponding operator is a full Hessian and hence the equation would be overdetermined. Instead we first use a hole-filling argument to show power decay for the $L^{p}$ integral of the second derivatives, and derive $L^{2}$ bounds for the third derivatives by a difference-quotient argument; these steps follow closely the ideas of Morrey [16] and L. Simon [19. In the final critical step we adapt a Gehring type lemma due to Bildhauer, Fuchs and Zhong [5] as well as the Moser-Trudinger inequality to get that the solution is of class $C^{2}$. Since it is also not immediate how to modify the $\mathcal{E}^{p}(f)$ approach to cover the functional $\mathcal{W}^{p}(f)$, we decided to include both independent arguments.

As second issue we address the existence of minimizers for the functionals. By the compactness theorem of Langer 14, sequences of closed immersed surfaces $f_{k}: \Sigma \rightarrow \mathbb{R}^{n}$ with $\mathcal{E}^{p}\left(f_{k}\right) \leq C$ subconverge weakly to an $f \in W_{\mathrm{im}}^{2, p}\left(\Sigma, \mathbb{R}^{n}\right)$, after suitable reparametrization and translation. In particular, we obtain the existence of a smooth $\mathcal{E}^{p}$ minimizer in the class of immersions $f: \Sigma \rightarrow \mathbb{R}^{n}$ for $p>2$. On the other hand, boundedness of $\mathcal{W}^{p}(f)$ is not sufficient to guarantee the required compactness. This is easily illustrated by joining two round spheres by a shrinking catenoid neck, showing that the $8 \pi$ bound in the following result is optimal.

Theorem 1.2. Let $\Sigma$ be a closed surface and $f_{k} \in W_{\mathrm{im}}^{2, p}\left(\Sigma, \mathbb{R}^{n}\right)$ be a sequence of immersions with $0 \in f_{k}(\Sigma)$ and

$$
\mathcal{W}^{p}\left(f_{k}\right) \leq C \quad \text { and } \quad \liminf _{k \rightarrow \infty} \frac{1}{4} \int_{\Sigma}\left|H_{k}\right|^{2} d \mu_{g_{k}}<8 \pi .
$$

After passing to $f_{k} \circ \varphi_{k}$ for appropriate $\varphi_{k} \in C^{\infty}(\Sigma, \Sigma)$ and selecting a subsequence, the $f_{k}$ converge weakly in $W^{2, p}\left(\Sigma, \mathbb{R}^{k}\right)$ to an $f \in W_{\mathrm{im}}^{2, p}\left(\Sigma, \mathbb{R}^{n}\right)$. In particular, the convergence is in $C^{1, \beta}\left(\Sigma, \mathbb{R}^{n}\right)$ for any $\beta<1-\frac{2}{p}$ and we have

$$
\mathcal{W}^{p}(f) \leq \liminf _{k \rightarrow \infty} \mathcal{W}^{p}\left(f_{k}\right)
$$

A classical approach to the construction of harmonic maps, due to Sacks \& Uhlenbeck [18, is by introducing perturbed functionals involving a power $p>2$ of the gradient. One motivation for our analysis is an analogous approximation for the Willmore functional

$$
\mathcal{W}(f)=\frac{1}{4} \int_{\Sigma}|H|^{2} d \mu_{g}=\frac{1}{4} \int_{\Sigma}|A|^{2} d \mu_{g}+\pi \chi(\Sigma) .
$$

The Willmore functional does not satisfy a Palais-Smale type condition, since it is invariant under the group of Möbius transformations. In Section 5 we verify suitable versions of the Palais-Smale condition for the functionals $\mathcal{E}^{p}$ and $\mathcal{W}^{p}$ with $p>2$. In a forthcoming paper, we study the limit $p \searrow 2$ in the case of $\mathcal{E}^{p}(f)$, proving a concentration compactness alternative and a partial blowup analysis.

Curvature functionals with nonquadratic growth appear also in the work of Bellettini, Dal Maso and Paolini [3] as well as Ambrosio and Masnou 1. However their focus is much different, for instance the latter paper is motivated by applications to image restoration. 


\section{The Euler Lagrange Equations}

Here we compute in local coordinates the Euler Lagrange equations of the functionals $\mathcal{E}^{p}(f)$ and $\mathcal{W}^{p}(f)$. For an immersed surface the fundamental forms are

$$
g_{\alpha \beta}=\left\langle\partial_{\alpha} f, \partial_{\beta} f\right\rangle \quad \text { and } \quad A_{\alpha \beta}=P^{\perp}\left(\partial_{\alpha \beta}^{2} f\right) .
$$

Here $P^{\perp}$ is the projection onto the normal space given by

$$
P^{\perp}=\mathrm{Id}-g^{\alpha \beta}\left\langle\partial_{\alpha} f, \cdot\right\rangle \partial_{\beta} f .
$$

We compute further

$$
|A|^{2}=g^{\alpha \gamma} g^{\beta \lambda}\left\langle P^{\perp} \partial_{\alpha \beta}^{2} f, \partial_{\gamma \lambda}^{2} f\right\rangle \quad \text { and } \quad|H|^{2}=g^{\alpha \beta} g^{\gamma \lambda}\left\langle P^{\perp} \partial_{\alpha \beta}^{2} f, \partial_{\gamma \lambda}^{2} f\right\rangle .
$$

On the open set of $W^{2, p}$ immersions, both $\mathcal{E}^{p}$ and $\mathcal{W}^{p}$ are differentiable in the sense of Fréchet. The derivative of $\mathcal{E}^{p}$ is given by

$$
\begin{aligned}
D \mathcal{E}^{p}(f) \phi & =\frac{p}{4} \int_{\Sigma}\left(1+|A|^{2}\right)^{\frac{p-2}{2}} g^{\alpha \gamma} g^{\beta \lambda}\left\langle P^{\perp} \partial_{\alpha \beta}^{2} f, \partial_{\gamma \lambda}^{2} \phi\right\rangle \sqrt{\operatorname{det} g} \\
& +\frac{p}{8} \int_{\Omega}\left(1+|A|^{2}\right)^{\frac{p-2}{2}}\left\langle\frac{\partial\left(g^{\alpha \gamma} g^{\beta \lambda} P^{\perp}\right)}{\partial p_{\mu}^{k}} \partial_{\mu} \phi^{k} \partial_{\alpha \beta}^{2} f, \partial_{\gamma \lambda}^{2} f\right\rangle \sqrt{\operatorname{det} g} \\
& +\frac{1}{4} \int_{\Omega}\left(1+|A|^{2}\right)^{\frac{p}{2}} \frac{\partial \sqrt{\operatorname{det} g}}{\partial p_{\mu}^{k}} \partial_{\mu} \phi^{k} .
\end{aligned}
$$

In particular if $f(x)=(x, u(x))$ where $u \in W^{2, p}\left(\Omega, \mathbb{R}^{n-2}\right)$, then $f$ is a critical point of $\mathcal{E}^{p}$ if and only if $u$ is a weak solution of the system

$$
\partial_{\alpha \beta}^{2}\left(a_{i}^{\alpha \beta}\left(D u, D^{2} u\right)\right)+\partial_{\alpha}\left(b_{i}^{\alpha}\left(D u, D^{2} u\right)\right)=0 \quad(1 \leq i \leq n-2),
$$

where the coefficients are given by

$$
\begin{aligned}
& a_{i}^{\alpha \beta}\left(D u, D^{2} u\right)=\left(1+|A|^{2}\right)^{\frac{p-2}{2}} \sqrt{\operatorname{det} g} g^{\alpha \gamma} g^{\beta \lambda}\left(\delta_{i j}-g^{\mu \nu} \partial_{\mu} u^{i} \partial_{\nu} u^{j}\right) \partial_{\gamma \lambda}^{2} u^{j} \\
& b_{i}^{\alpha}\left(D u, D^{2} u\right)= \\
& \quad-\frac{1}{2}\left(1+|A|^{2}\right)^{\frac{p-2}{2}} \frac{\partial\left(g^{\gamma \mu} g^{\lambda \nu}\left(\delta_{j k}-g^{\sigma \tau} p_{\sigma}^{j} p_{\tau}^{k}\right)\right)}{\partial p_{\alpha}^{i}} \partial_{\gamma \lambda}^{2} u^{j} \partial_{\mu \nu}^{2} u^{k} \sqrt{\operatorname{det} g} \\
& \quad-\frac{1}{p}\left(1+|A|^{2}\right)^{\frac{p}{2}} \frac{\partial \sqrt{\operatorname{det} g}}{\partial p_{\alpha}^{i}} .
\end{aligned}
$$

For $|p| \leq \Lambda$ and $V=\left(1+|q|^{2}\right)^{\frac{1}{2}}$, where $p, q$ are the variables corresponding to $D u, D^{2} u$, one easily checks the bounds

$$
\begin{aligned}
\left|D_{q} a\right| & \leq C(\Lambda) V^{p-2} \\
|a|+\left|D_{p} a\right|+\left|D_{q} b\right| & \leq C(\Lambda) V^{p-1} \\
|b|+\left|D_{p} b\right| & \leq C(\Lambda) V^{p} .
\end{aligned}
$$

Moreover, the system satisfies the ellipticity condition

$$
\frac{\partial a_{i}^{\alpha \beta}}{\partial q_{\gamma \lambda}^{j}} \xi_{\alpha \beta}^{i} \xi_{\gamma \lambda}^{j} \geq \lambda V^{p-2}|\xi|^{2} \quad \text { where } \lambda=\lambda(\Lambda)>0,
$$


For the first variation of $\mathcal{W}^{p}(f)$ one obtains

$$
\begin{aligned}
D \mathcal{W}^{p}(f) \phi & =\frac{p}{4} \int_{\Sigma}\left(1+|H|^{2}\right)^{\frac{p-2}{2}}\left\langle H, g^{\gamma \lambda} \partial_{\gamma \lambda}^{2} \phi\right\rangle \sqrt{\operatorname{det} g} \\
& +\frac{p}{8} \int_{\Omega}\left(1+|H|^{2}\right)^{\frac{p-2}{2}}\left\langle\frac{\partial\left(g^{\alpha \beta} g^{\gamma \lambda} P^{\perp}\right)}{\partial p_{\mu}^{k}} \partial_{\mu} \phi^{k} \partial_{\alpha \beta}^{2} f, \partial_{\gamma \lambda}^{2} f\right\rangle \sqrt{\operatorname{det} g} \\
& +\frac{1}{4} \int_{\Omega}\left(1+|H|^{2}\right)^{\frac{p}{2}} \frac{\partial \sqrt{\operatorname{det} g}}{\partial p_{\mu}^{k}} \partial_{\mu} \phi^{k} .
\end{aligned}
$$

Putting $L_{g} \phi=\sqrt{\operatorname{det} g} g^{\gamma \lambda} \partial_{\gamma \lambda}^{2} \phi$ the first variation takes the form

$$
D \mathcal{W}^{p}(f) \phi=\frac{p}{4} \int_{\Omega}\left(1+|H|^{2}\right)^{\frac{p-2}{2}}\left\langle H, L_{g} \phi\right\rangle+\int_{\Omega} B_{i}^{\alpha}\left(D f, D^{2} f\right) \partial_{\alpha} \phi^{i},
$$

where

$$
\begin{aligned}
B_{i}^{\alpha}\left(D f, D^{2} f\right)= & \frac{p}{8}\left(1+|H|^{2}\right)^{\frac{p-2}{2}}\left\langle\frac{\partial\left(g^{\gamma \lambda} g^{\mu \nu} P^{\perp}\right)}{\partial p_{\alpha}^{i}} \partial_{\gamma \lambda}^{2} f, \partial_{\mu \nu}^{2} f\right\rangle \sqrt{\operatorname{det} g} \\
& +\frac{1}{4}\left(1+|H|^{2}\right)^{\frac{p}{2}} \frac{\partial \sqrt{\operatorname{det} g}}{\partial p_{\alpha}^{i}} .
\end{aligned}
$$

When passing to graphs we have under the assumption $|p| \leq \Lambda$

$$
|B|+\left|D_{p} B\right| \leq C(\Lambda) V^{p-2}|q|^{2} \quad \text { and } \quad\left|D_{q} B\right| \leq C(\Lambda) V^{p-2}|q| .
$$

\section{Regularity of CRitical POINTS}

3.1. The functional $\mathcal{W}^{p}$. For $\Omega \subset \mathbb{R}^{2}$ and $p>2$, let $f: \Omega \rightarrow \mathbb{R}^{n}$ be the graph of a function $u \in W^{2, p}\left(\Omega, \mathbb{R}^{n-2}\right)$. Recall from (2.3) that $f$ is a critical point of $\mathcal{W}^{p}$ if and only if

$$
\int_{\Omega}\left\langle\mathcal{H}, L_{g} \varphi\right\rangle+\int_{\Omega} B_{i}^{\alpha}\left(D u, D^{2} u\right) \partial_{\alpha} \varphi^{i}=0 \quad \text { for all } \varphi \in W_{0}^{2, p}\left(\Omega, \mathbb{R}^{n}\right) .
$$

Here $\mathcal{H}=\left(1+|H|^{2}\right)^{\frac{p}{2}-1} H$ and the functions $B_{\alpha}^{i}$ satisfy the bounds (2.4). We have the following result.

Theorem 3.1. Weak solutions $u \in W^{2, p}\left(\Omega, \mathbb{R}^{n-2}\right)$ of (3.1) are smooth.

3.1.1. $W^{2, q}$-regularity. We start by stating a regularity property for the mean curvature system. For a graph of a function $u \in W^{2, p}\left(\Omega, \mathbb{R}^{n-2}\right)$, the weak mean curvature satisfies for $j=1, \ldots, n-2$ the formula

$$
g^{\alpha \beta}\left(\delta_{i j}-g^{\lambda \mu} \partial_{\lambda} u^{i} \partial_{\mu} u^{j}\right) \partial_{\alpha \beta}^{2} u^{i}=H^{j+2} .
$$

Since $p>2$ the left hand side may be viewed as a linear operator of the form $a_{i j}^{\alpha \beta} \partial_{\alpha \beta}^{2} u^{i}$, where the coefficients are Hölder continuos with exponent $1-\frac{2}{p}>0$ and the ellipticity constant is controlled by the $W^{2, p}$-norm of the function $u$. In particular, if we know $H \in L^{q}\left(\Omega, \mathbb{R}^{n}\right)$ for some $q \in(p, \infty)$, then standard $L^{q}$ theory yields $u \in W_{l o c}^{2, q}\left(\Omega, \mathbb{R}^{n-2}\right)$ together with a local estimate

$$
\|u\|_{W^{2, q}\left(\Omega^{\prime}\right)} \leq C(p, q, \Lambda)\left(\|H\|_{L^{q}(\Omega)}+1\right) \quad \text { if }\|u\|_{W^{2, p}(\Omega)} \leq \Lambda .
$$

The dependence on the domains $\Omega^{\prime} \subset \Omega$ is not mentioned explicitely here. 
Lemma 3.2. Let $u \in W^{2, p}\left(\Omega, \mathbb{R}^{n-2}\right)$ be a weak solution of (3.1). Then for any $\varphi \in W^{2, p}(\Omega)$ and any test function $\eta \in C_{0}^{2}(\Omega)$ we have

$$
\int_{\Omega} \eta\left\langle\mathcal{H}, L_{g} \varphi\right\rangle \leq C \int_{\operatorname{spt} \eta}\left(1+\left|D^{2} u\right|^{2}\right)^{\frac{p}{2}}(|\varphi|+|D \varphi|), \quad \text { where } C=C\left(\|\eta\|_{C^{2}}\right) .
$$

Proof. Expanding

$$
L_{g}(\eta \varphi)=\eta L_{g} \varphi+\varphi L_{g} \eta+2 \sqrt{\operatorname{det} g} g^{\alpha \beta} \partial_{\alpha} \eta \partial_{\beta} \varphi
$$

we see by combining with (3.1) and (2.4) that

$$
\begin{aligned}
\int_{\Omega} \eta\left\langle\mathcal{H}, L_{g} \varphi\right\rangle \leq & C \int_{\Omega}\left(1+\left|D^{2} u\right|^{2}\right)^{\frac{p}{2}}(|D \eta||\varphi|+|\eta||D \varphi|) \\
& +C \int_{\Omega}\left(1+\left|D^{2} u\right|^{2}\right)^{\frac{p-1}{2}}\left(\left|D^{2} \eta\right||\varphi|+|D \eta||D \varphi|\right) .
\end{aligned}
$$

This implies the lemma.

We are now ready to improve the integrability of $D^{2} u$.

Theorem 3.3. Let $u \in W^{2, p}\left(\Omega, \mathbb{R}^{n-2}\right)$ be a weak solution of (3.1) where $p>2$. Then $u \in W_{l o c}^{2, q}\left(\Omega, \mathbb{R}^{n-2}\right)$ for any $q \in[p, \infty)$.

Proof. Assume we know already $\|u\|_{W^{2, q}\left(B_{r}\right)} \leq \Lambda$ where $q \geq p$. For $|H|_{A}=$ $\min (|H|, A)$ with $A>0$ and a parameter $\lambda \in(1, q)$, we use $L^{q}$-theory to obtain a solution $\varphi \in W^{2, q} \cap W_{0}^{1, q}\left(B_{r}, \mathbb{R}^{n}\right)$ of the linear equation

$$
L_{g} \varphi=|H|_{A}^{\lambda-1} H \text {. }
$$

As $1<\frac{q}{\lambda}<\infty$ the function $\varphi$ satisfies

$$
\|\varphi\|_{W^{2, \frac{q}{\lambda}\left(B_{r}\right)}} \leq C\left|\left\|\left.H\right|_{A} ^{\lambda-1} H\right\|_{L^{\frac{q}{\lambda}\left(B_{r}\right)}} \leq C(\Lambda)\right.
$$

By the Sobolev embedding theorem, we have for $\lambda<\frac{q}{2}$ the estimate

$$
\|\varphi\|_{C^{1}\left(B_{r}\right)} \leq C(\Lambda)
$$

while for $\frac{q}{2}<\lambda<q$ we get instead

$$
\|\varphi\|_{W^{1, s}\left(B_{r}\right)} \leq C(\Lambda) \quad \text { for } s=\frac{2 q}{2 \lambda-q} \in[1, \infty)
$$

Now Lemma 3.2 implies that

$$
\int_{B_{\frac{r}{2}}}|H|^{p}|H|_{A}^{\lambda-1} \leq C \int_{B_{r}}\left(1+\left|D^{2} u\right|^{2}\right)^{\frac{p}{2}}(|\varphi|+|D \varphi|) \leq C(\Lambda),
$$

under the condition that either $1<\lambda<\frac{q}{2}$, or that $\frac{q}{2}<\lambda<q$ with

$$
\frac{p}{q}+\frac{1}{s} \leq 1 \quad \Leftrightarrow \quad \lambda \leq \frac{3 q}{2}-p
$$

Letting $A \nearrow \infty$ we get $H \in L^{p+\lambda-1}\left(B_{\frac{r}{2}}\right)$, and then obtain $u \in W^{2, p+\lambda-1}\left(B_{\frac{r}{4}}, \mathbb{R}^{n-2}\right)$ from (3.3). We can now set up an iteration to get $u \in W_{l o c}^{2, q}(\Omega)$ for all $q<\infty$. As initial step we choose $q=p$ and $1<\lambda<\frac{p}{2}$, which brings us to $q<\frac{3 p}{2}-1$. For $p<q<2 p$ we can take $\lambda=\frac{3 q}{2}-p$, improving the exponent to $\frac{3 q}{2}-1$. After finitely many iterations, we arrive at some $q>2 p$. Now we continue with $\frac{q}{2}<\lambda=q-p+2<\frac{3 q}{2}-p$ and obtain the desired higher integrability. 
3.1.2. $W_{\text {loc }}^{1,2}$-Regularity of $\mathcal{H}$. In this subsection we use difference quotient methods in order to show that $\mathcal{H} \in W_{l o c}^{1,2}$. For $h>0, f: \Omega \rightarrow \mathbb{R}^{k}$ and fixed $\nu \in\{1,2\}$ we define

$$
f_{h}(x)=\frac{1}{h}\left(f\left(x+h e_{\nu}\right)-f(x)\right)
$$

In the first Lemma we compare the difference quotients of $\mathcal{H}$ with the ones of $H$ and $|H|^{2}$.

Lemma 3.4. Let $u$ be as in Theorem 3.1 and let $\Omega^{\prime} \subset \Omega$. Then, for every $1 \leq q<\infty$ and for all $h>0$ small enough we have for all $x \in \Omega^{\prime}$

$$
\left|\left(|H|^{2}\right)_{h}\right|(x)+\left|H_{h}\right|(x) \leq \Phi(x, h)\left|\mathcal{H}_{h}\right|(x),
$$

where

$$
\int_{B_{r}}|\Phi(x, h)|^{q} d x \leq C(q)
$$

Proof. We have $|\mathcal{H}|^{2}=\left(1+|H|^{2}\right)^{p-2}|H|^{2}$ and we get from the mean value theorem

$$
\begin{aligned}
\left(|\mathcal{H}|^{2}\right)_{h}(x) & =\left(\left(1+|H|^{2}\right)^{p-2}\right)_{h}(x)|H|^{2}\left(x+h e_{\nu}\right)+\left(1+|H|^{2}\right)^{p-2}(x)\left(|H|^{2}\right)_{h}(x) \\
& =\left(|H|^{2}\right)_{h}(x)\left[(p-2)(1+\xi(x))^{p-3}|H|^{2}\left(x+h e_{v}\right)+\left(1+|H|^{2}\right)^{p-2}(x)\right],
\end{aligned}
$$

where $0 \leq \xi(x) \leq|H|^{2}(x)+|H|^{2}\left(x+h e_{\nu}\right)$. Hence we have

$$
\left|\left(|H|^{2}\right)_{h}\right|(x) \leq\left|\left(|\mathcal{H}|^{2}\right)_{h}\right|(x)
$$

On the other hand we calculate

$$
\left(|\mathcal{H}|^{2}\right)_{h}(x)=(\mathcal{H})_{h}(x) \mathcal{H}\left(x+h e_{\nu}\right)+(\mathcal{H})_{h}(x) \mathcal{H}(x) .
$$

Combining this with Theorem 3.3 proves the first estimate.

Another application of the mean value theorem yields

$$
\mathcal{H}_{h}(x)=H_{h}(x)\left(1+|H|^{2}\right)^{\frac{p}{2}-1}\left(x+h e_{\nu}\right)+\left(\frac{p}{2}-1\right)(1+\xi(x))^{\frac{p}{2}-2}\left(|H|^{2}\right)_{h}(x) H(x),
$$

where $0 \leq \xi(x) \leq|H|^{2}+|H|_{h}^{2}$ and hence the second estimate follows from the first one.

We have the following Corollary.

Corollary 3.5. Let $u$ be as in Theorem 3.1 and let $B_{r} \subset \Omega$. Then, for every $1<s<\infty$ and for all $h>0$ small enough we have

$$
\int_{B_{r}} \eta^{2 s}\left|\left(D^{2} u\right)_{h}\right|^{s} \leq C \int_{B_{r}} \Phi(x, h)\left(\eta^{2 s}\left|(\mathcal{H})_{h}\right|^{s}+1\right),
$$

where $\eta \in C_{c}^{\infty}\left(B_{r}\right)$ is a smooth cut-off function and $\Phi$ satisfies

$$
\int_{B_{r}}|\Phi(x, h)|^{q} d x \leq C(q) \quad \forall 1 \leq q<\infty .
$$

Proof. Using (3.2) we see that $u_{h}$ solves

$$
a_{i j}^{\alpha \beta}\left(\cdot+h e_{\nu}\right) \partial_{\alpha \beta}^{2} u_{h}^{i}=H_{h}^{j+2}-\left(a_{i j}^{\alpha \beta}\right)_{h} \partial_{\alpha \beta}^{2} u^{i}=: \tilde{H}^{j}(x),
$$

where $a_{i j}^{\alpha \beta}=g^{\alpha \beta}\left(\delta_{i j}-g^{\lambda \mu} \partial_{\lambda} u^{i} \partial_{\mu} u^{j}\right)$. 
Using Theorem 3.3 and Lemma 3.4, a standard estimate shows that there exists a function $\Phi_{1}(x, h)$ satisfying

$$
\int_{B_{r}}\left|\Phi_{1}(x, h)\right|^{q} d x \leq C(q),
$$

for all $1<q<\infty$ and all $h>0$ small, such that

$$
|\tilde{H}|(x) \leq \Phi_{1}(x, h)\left(\left|\mathcal{H}_{h}\right|+1\right) .
$$

Next we use standard $L^{p}$-theory in order to get for every $1<s<\infty$

$$
\begin{aligned}
\int_{B_{r}}\left|D^{2}\left(\eta^{2} u_{h}\right)\right|^{s} & \leq C \int_{B_{r}}\left(\eta^{2 s}|\tilde{H}|^{s}+\left|D u_{h}\right|^{s}+\left|u_{h}\right|^{s}\right) \\
& \leq C \int_{B_{r}} \Phi_{2}(x, h)\left(\left|\mathcal{H}_{h}\right|^{s}+1\right),
\end{aligned}
$$

where $\Phi_{2}(x, h)$ satisfies

$$
\int_{B_{r}}\left|\Phi_{2}(x, h)\right|^{q} d x \leq C(q),
$$

for all $h>0$ small and all $1<q<\infty$.

Since moreover

$$
\begin{aligned}
\int_{B_{r}} \eta^{2 s}\left|\left(D^{2} u\right)_{h}\right|^{s} & \leq C \int_{B_{r}}\left(\left|D^{2}\left(\eta^{2} u_{h}\right)\right|^{s}+\left|D\left(\eta^{2} u_{h}\right)\right|^{s}+\left|\eta^{2} u_{h}\right|^{s}\right) \\
& \leq C \int_{B_{r}}\left|D^{2}\left(\eta^{2} u_{h}\right)\right|^{s}+C \int_{B_{r}} \Phi_{3}(x, h),
\end{aligned}
$$

for some function $\Phi_{3}$ with the same properties as $\Phi_{1}$ and $\Phi_{2}$, this finishes the proof of the Corollary.

Now we are in a position to prove that $\mathcal{H} \in W_{\text {loc }}^{1,2}(\Omega)$.

Proposition 3.6. Let $u \in W^{2, p}\left(\Omega, \mathbb{R}^{n-2}\right)$ be as in Theorem 3.1. Then we have that $\mathcal{H} \in W_{\text {loc }}^{1,2}\left(\Omega, \mathbb{R}^{n}\right)$.

Proof. Taking difference quotients of equation (3.1) we get

$$
\partial_{\alpha \beta}^{2}\left(\sqrt{g} g^{\alpha \beta} \mathcal{H}\right)_{h}-\partial_{\alpha}\left(B^{\alpha}\left(D u, D^{2} u\right)\right)_{h}=0 .
$$

We abbreviate $U(x)=\left(D u(x), D^{2} u(x)\right)$ and we use the fundamental theorem of Calculus to write

$$
\begin{aligned}
(f \circ U)_{h}(x) & =\frac{1}{h}\left(f\left(U\left(x+h e_{\nu}\right)\right)-f(U(x))\right) \\
& =\frac{1}{h} \int_{0}^{1} \frac{d}{d t} f\left((1-t) U(x)+t U\left(x+h e_{\nu}\right)\right) d t \\
& =\int_{0}^{1} D f\left((1-t) U(x)+t U\left(x+h e_{\nu}\right)\right) d t \cdot U_{h}(x) .
\end{aligned}
$$

Using the notation $f^{h}(x)=\int_{0}^{1} f\left((1-t) U(x)+t U\left(x+h e_{\nu}\right)\right) d t$ we thus get

$$
(f \circ U)_{h}=\left(\frac{\partial f}{\partial q_{\lambda \mu}^{j}}\right)^{h} \partial_{\lambda \mu}^{2} u_{h}^{j}+\left(\frac{\partial f}{\partial p_{\lambda}^{j}}\right)^{h} \partial_{\lambda} u_{h}^{j},
$$


and the system (3.6) takes the form

$$
\partial_{\alpha \beta}^{2}\left(\left(\sqrt{g} g^{\alpha \beta}\right)\left(x+h e_{\nu}\right) \mathcal{H}_{h}\right)+\partial_{\alpha \beta}^{2}\left(\left(\sqrt{g} g^{\alpha \beta}\right)_{h} \mathcal{H}\right)-\partial_{\alpha}\left(\tilde{B}^{\alpha}\left(D u_{h}, D^{2} u_{h}\right)\right)=0
$$

Here the coefficients are given as follows:

$$
\tilde{B}^{\alpha}(x, z, p, q)=\left(\frac{\partial B^{\alpha}}{\partial q_{\lambda \mu}^{j}}\right)^{h}(x) q_{\lambda \mu}^{j}+\left(\frac{\partial B^{\alpha}}{\partial p_{\lambda}^{j}}\right)^{h}(x) p_{\lambda}^{j} .
$$

In order to state bounds for these coefficients, we introduce the abbreviation

$$
I_{s, h}(x)=\int_{0}^{1}\left(1+\left|(1-t) D^{2} u(x)+t D^{2} u(x+h)\right|^{2}\right)^{s / 2} d t .
$$

Using (2.4) we then obtain

$$
|\tilde{B}(p, q)| \leq C\left(I_{p-1, h}(x)|q|+I_{p, h}(x)|p|\right) .
$$

For $\|D u\|_{L^{\infty}}<\infty$ we get

$$
\left|\left(\sqrt{g} g^{-1}\right)_{h} \mathcal{H}\right|(x) \leq C\left(1+\left|D^{2} u\right|^{2}\right)^{\frac{p-1}{2}}(x)\left|D u_{h}\right|(x)
$$

and moreover the operator

$$
\tilde{L} v(x):=\left(\sqrt{g} g^{\alpha \beta}\right)\left(x+h e_{\nu}\right) \partial_{\alpha \beta}^{2} v(x)
$$

is strongly elliptic. We let $B_{r} \subset \Omega$ and by standard $L^{p}$-theory there exists a solution $\tilde{\varphi} \in W^{2,4} \cap W_{0}^{1,4}\left(B_{r}, \mathbb{R}^{n}\right)$ of

satisfying

$$
\tilde{L} \tilde{\varphi}=\mathcal{H}
$$

$$
\|\tilde{\varphi}\|_{W^{2,4}\left(B_{r}\right)} \leq C\|\mathcal{H}\|_{L^{4}\left(B_{r}\right)} .
$$

Next we let $\eta \in C_{c}^{\infty}\left(B_{r}\right)$ be a smooth cut-off function and we define $\varphi_{h}=\eta^{4} \tilde{\varphi}_{h}$. A standard computation then shows that

$$
\begin{aligned}
\tilde{L} \varphi_{h}= & \eta^{4} \mathcal{H}_{h}-\left(\sqrt{g} g^{\alpha \beta}\right)_{h}\left(x+h e_{\nu}\right) \partial_{\alpha \beta}^{2}\left(\eta^{4} \tilde{\varphi}\right)\left(x+h e_{\nu}\right) \\
& +4 \eta^{2}\left(\eta\left(\sqrt{g} g^{\alpha \beta}\right)\left(x+h e_{\nu}\right) \partial_{\alpha} \eta \partial_{\beta} \tilde{\varphi}_{h}\right. \\
& \left.+\eta\left(\sqrt{g} g^{\alpha \beta}\right)\left(x+h e_{\nu}\right) \partial_{\alpha \beta}^{2} \eta \tilde{\varphi}_{h}+3\left(\sqrt{g} g^{\alpha \beta}\right)\left(x+h e_{\nu}\right) \partial_{\alpha} \eta \partial_{\beta} \eta \tilde{\varphi}_{h}\right) \\
= & \eta^{4} \mathcal{H}_{h}+\eta^{2} R\left[\tilde{\varphi}, D \tilde{\varphi}, D^{2} \tilde{\varphi}\right]
\end{aligned}
$$

and, by using standard $L^{2}$-estimates, we conclude

$$
\begin{aligned}
\left\|\varphi_{h}\right\|_{W^{2,2}\left(B_{r}\right)} & \leq C(r)\left(\left\|\eta^{2} \mathcal{H}_{h}\right\|_{L^{2}\left(B_{r}\right)}+\left\|\eta^{2} \tilde{\varphi}_{h}\right\|_{W^{1,2}\left(B_{r}\right)}+\left\|R\left[\tilde{\varphi}, D \tilde{\varphi}, D^{2} \tilde{\varphi}\right]\right\|_{L^{2}\left(B_{r}\right)}\right) \\
& \leq C(r)\left(\left\|\eta^{2} \mathcal{H}_{h}\right\|_{L^{2}\left(B_{r}\right)}+\left(\int_{B_{r}} \Phi(x, h) d x\right)^{\frac{1}{2}}\right),
\end{aligned}
$$

where here and in the following we let $\Phi(x, h)$ be a function satisfying

$$
\int_{B_{r}}|\Phi(x, h)|^{q} d x \leq C(q) \quad \forall 1 \leq q<\infty .
$$

Sobolev's embedding theorem then gives for every $q<\infty$

$$
\|\varphi\|_{W^{1, q}\left(B_{r}\right)} \leq C(r)\left(\left\|\eta^{2} \mathcal{H}_{h}\right\|_{L^{2}\left(B_{r}\right)}+\left(\int_{B_{r}} \Phi(x, h) d x\right)^{\frac{1}{2}}\right) .
$$


Using $\varphi_{h}$ as a test function in (3.7) we conclude

$$
\begin{aligned}
\int_{B_{r}} \eta^{4}\left|\mathcal{H}_{h}\right|^{2} \leq & C \int_{B_{r}}\left(\left|\left(\sqrt{g} g^{-1}\right)_{h} \mathcal{H}\right|\left|D^{2} \varphi_{h}\right|+\left|\tilde{B}\left(D u_{h}, D^{2} u_{h}\right)\right|\left|D \varphi_{h}\right|\right. \\
& \left.+\eta^{2}\left|\mathcal{H}_{h}\right|\left|R\left[\tilde{\varphi}, D \tilde{\varphi}, D^{2} \tilde{\varphi}\right]\right|\right) \\
= & I+I I+I I I .
\end{aligned}
$$

Next we estimate all three integrals separately. We start with $I$. Combining (3.9), (3.10), Theorem 3.3 and Hölder's inequality we conclude

$$
\begin{aligned}
I & \leq C\left\|D^{2} \varphi_{h}\right\|_{L^{2}\left(B_{r}\right)}\left(\int_{B_{r}}\left(1+\left|D^{2} u\right|^{2}\right)^{p-1}\left|D u_{h}\right|^{2}\right)^{\frac{1}{2}} \\
& \leq C\left(\int_{B_{r}} \Phi(x, h) d x\right)^{\frac{1}{2}}\left(\left\|\eta^{2} \mathcal{H}_{h}\right\|_{L^{2}\left(B_{r}\right)}+\left(\int_{B_{r}} \Phi(x, h) d x\right)^{\frac{1}{2}}\right) \\
& \leq \delta \int_{B_{r}} \eta^{4}\left|\mathcal{H}_{h}\right|^{2}+C \int_{B_{r}} \Phi(x, h) d x .
\end{aligned}
$$

Using Corollary 3.5, (3.8) and Hölder's inequality we get

$$
\begin{aligned}
I I \leq & C\left\|\tilde{\varphi}_{h}\right\|_{W^{1,4}\left(B_{r}\right)}\left(\int_{B_{r}} I_{p-1, h}^{\frac{4}{3}} \eta^{\frac{8}{3}}\left|D^{2} u_{h}\right|^{\frac{4}{3}}+I_{p, h}^{\frac{4}{3}}\left|D u_{h}\right|^{\frac{4}{3}}\right)^{\frac{3}{4}} \\
\leq & \left.C\left\|\tilde{\varphi}_{h}\right\|_{W^{1,4}\left(B_{r}\right)}\left(\left(\int_{B_{r}} \eta^{3}\left|D^{2} u_{h}\right|^{\frac{3}{2}}\right)^{\frac{2}{3}}\left(\int_{B_{r}} I_{p-1, h}^{12}\right)^{\frac{1}{12}}+\int_{B_{r}} \Phi(x, h)\right)^{\frac{3}{4}}\right) \\
\leq & C\left\|\tilde{\varphi}_{h}\right\|_{W^{1,4}\left(B_{r}\right)}\left(\left(\int_{B_{r}} \eta^{3} \Phi(x, h)\left|\mathcal{H}_{h}\right|^{\frac{3}{2}}+\Phi(x, h)\right)^{\frac{2}{3}}\right. \\
& \left.\cdot\left(\int_{B_{r}} \Phi(x, h)\right)^{\frac{1}{12}}+\left(\int_{B_{r}} \Phi(x, h)\right)^{\frac{3}{4}}\right) .
\end{aligned}
$$

Using the fact that $\tilde{\varphi} \in W^{2,4}\left(B_{r}\right)$ we get for $h>0$ small enough

$$
I I \leq \delta \int_{B_{r}} \eta^{4}\left|\mathcal{H}_{h}\right|^{2}+\int_{B_{r}} \Phi(x, h)
$$

Finally we get

$$
\begin{aligned}
I I I & \leq \delta \int_{B_{r}} \eta^{4}\left|\mathcal{H}_{h}\right|^{2}+\int_{B_{r}}\left|R\left[\tilde{\varphi}, D \tilde{\varphi}, D^{2} \tilde{\varphi}\right]\right|^{2} \\
& \leq \delta \int_{B_{r}} \eta^{4}\left|\mathcal{H}_{h}\right|^{2}+\int_{B_{r}} \Phi(x, h) .
\end{aligned}
$$

Combining all these estimates yields for $\delta$ small enough

$$
\int_{B_{r}} \eta^{4}\left|\mathcal{H}_{h}\right|^{2} \leq \int_{B_{r}} \Phi(x, h)
$$

and therefore we can let $h \rightarrow 0$ in order to conclude that $\mathcal{H} \in W_{\text {loc }}^{1,2}\left(\Omega, \mathbb{R}^{n}\right)$ with

$$
\int_{K} \eta^{4}|D \mathcal{H}|^{2} \leq C
$$

for all $K \subset \subset \Omega$.

Corollary 3.7. Let $u \in W^{2, p}\left(\Omega, \mathbb{R}^{n-2}\right)$ be as in Theorem 3.1. Then we have that $H \in W_{\text {loc }}^{1, s}\left(\Omega, \mathbb{R}^{n}\right)$ and $u \in W_{\text {loc }}^{3, s}\left(\Omega, \mathbb{R}^{n-2}\right)$ for all $s<2$. 
Proof. Combining Theorem 3.3 and Proposition 3.6 we get that

$$
|\mathcal{H}|^{2} \in W_{\text {loc }}^{1, s}(\Omega)
$$

for all $s<2$. Using the formulas

$$
D|\mathcal{H}|^{2}=D|H|^{2}\left(\left(1+|H|^{2}\right)^{p-2}+(p-2)|H|^{2}\left(1+|H|^{2}\right)^{p-3}\right)
$$

and

$$
\left(1+|H|^{2}\right)^{\frac{p-2}{2}} D H=D \mathcal{H}-\frac{p-2}{2}\left(1+|H|^{2}\right)^{\frac{p-4}{2}} H D|H|^{2},
$$

we conclude that

$$
|D H| \leq C\left(|D \mathcal{H}|+\left.|D| \mathcal{H}\right|^{2} \mid\right)
$$

Therefore we get that

$$
H \in W_{l o c}^{1, s}\left(\Omega, \mathbb{R}^{n}\right)
$$

for all $s<2$. Arguing as at the beginning of this subsection we conclude that

$$
u \in W_{l o c}^{3, s}\left(\Omega, \mathbb{R}^{n-2}\right)
$$

for all $s<2$.

3.1.3. Higher regularity. In this last subsection we show the higher regularity for solutions of (3.1). We start by showing that $\mathcal{H} \in W^{1,2+\gamma}\left(\Omega, \mathbb{R}^{n}\right)$ for some $0<\gamma<$ $\frac{1}{2}$.

In order to see this, we let $B_{r} \subset \Omega$ and we let $\varphi_{1} \in W^{2, \frac{2}{1+\gamma}} \cap W_{0}^{1, \frac{2}{1+\gamma}}\left(B_{r}, \mathbb{R}^{n}\right)$ be the solution of

$$
\tilde{L} \varphi_{1}=\left|\mathcal{H}_{h}\right|^{\gamma} \mathcal{H}_{h}
$$

satisfying

$$
\left\|\varphi_{1}\right\|_{W^{2, \frac{2}{1+\gamma}\left(B_{r}\right)}} \leq C\left\|\mathcal{H}_{h}\right\|_{L^{2}\left(B_{r}\right)}^{1+\gamma}
$$

Sobolev's embedding theorem yields

$$
\left\|\varphi_{1}\right\|_{L^{\infty}\left(B_{r}\right)}+\left\|D \varphi_{1}\right\|_{L^{\frac{2}{\gamma}\left(B_{r}\right)}} \leq C\left\|\mathcal{H}_{h}\right\|_{L^{2}\left(B_{r}\right)}^{1+\gamma} .
$$

Next we let $\eta \in C_{c}^{\infty}\left(B_{r}\right)$ and we define $\tilde{\varphi_{1}}=\eta^{4} \varphi_{1}$. We conclude that

$$
\begin{aligned}
\tilde{L} \tilde{\varphi_{1}}= & \eta^{4}\left|\mathcal{H}_{h}\right|^{\gamma} \mathcal{H}_{h}+8 \eta^{3}\left(\sqrt{g} g^{\alpha \beta}\right)\left(x+h e_{\nu}\right) \partial_{\alpha} \eta \partial_{\beta} \varphi_{1} \\
& +4 \eta^{2} \varphi_{1}\left(\sqrt{g} g^{\alpha \beta}\right)\left(x+h e_{\nu}\right)\left(3 \partial_{\alpha} \eta \partial_{\beta} \eta+\eta \partial_{\alpha \beta}^{2} \eta\right) \\
= & : \eta^{4}\left|\mathcal{H}_{h}\right|^{\gamma} \mathcal{H}_{h}+\eta^{2} L\left[\varphi_{1}, D \varphi_{1}\right] .
\end{aligned}
$$

Moreover we have that

$$
\left\|\tilde{\varphi}_{1}\right\|_{L^{\infty}\left(B_{r}\right)}+\left\|D \tilde{\varphi_{1}}\right\|_{L^{\frac{2}{\gamma}\left(B_{r}\right)}}+\left\|D^{2} \tilde{\varphi}_{1}\right\|_{L^{\frac{2}{1+\gamma}\left(B_{r}\right)}} \leq C\left\|\mathcal{H}_{h}\right\|_{L^{2}\left(B_{r}\right)}^{1+\gamma} .
$$

Now we use $\tilde{\varphi}_{1}$ as a test function in equation (3.7) and we conclude

$$
\begin{aligned}
\int_{B_{r}} \eta^{4}\left|\mathcal{H}_{h}\right|^{2+\gamma} \leq & C \int_{B_{r}}\left(\left|\left(\sqrt{g} g^{-1}\right)_{h} \mathcal{H}\right|\left|D^{2} \tilde{\varphi}_{1}\right|+\left|\tilde{B}\left(D u_{h}, D^{2} u_{h}\right)\right|\left|D \tilde{\varphi}_{1}\right|\right. \\
& \left.+\eta^{2}\left|\mathcal{H}_{h}\right|\left|L\left[\varphi_{1}, D \varphi_{1}\right]\right|\right) \\
= & i)+i i)+i i i) .
\end{aligned}
$$


As before we estimate the three integrals separately. Using (3.9) and Hölder's inequality we get for $h$ small enough

$$
\begin{aligned}
i) & \leq C|| D^{2} \tilde{\varphi}_{1}\left\|_{L^{\frac{2}{1+\gamma}}\left(B_{r}\right)}\right\|\left(1+\left|D^{2} u\right|^{2}\right)^{\frac{p-1}{2}}\left|D u_{h}\right| \|_{L^{\frac{2}{1-\gamma}}}\left(B_{r}\right) \\
& \leq C .
\end{aligned}
$$

Corollary 3.7 and (3.8) imply for $h$ small enough

$$
\begin{aligned}
i i) \leq & C\left\|D \tilde{\varphi_{1}}\right\|_{L^{\frac{2}{\gamma}\left(B_{r}\right)}}\left(\left\|D^{2} u_{h}\right\|_{L^{\frac{4}{3}}\left(B_{r}\right)}\left\|I_{p-1, h}\right\|_{L^{\frac{4}{1-2 \gamma}}\left(B_{r}\right)}\right. \\
& \left.+\left\|D u_{h}\right\|_{L^{\frac{4}{3}}\left(B_{r}\right)}\left\|I_{p, h}\right\|_{L^{\frac{4}{1-2 \gamma}}\left(B_{r}\right)}\right) \\
\leq & C .
\end{aligned}
$$

Finally we have

$$
\text { iii) } \leq C\left\|\mathcal{H}_{h}\right\|_{L^{2}\left(B_{r}\right)}\left\|\varphi_{1}\right\|_{W^{1,2}\left(B_{r}\right)} \leq C .
$$

Combining thes estimates we conclude

$$
\int_{B_{\frac{r}{2}}}\left|\mathcal{H}_{h}\right|^{2+\gamma} \leq C
$$

and therefore we get that

$$
\mathcal{H} \in W_{l o c}^{1,2+\gamma}\left(\Omega, \mathbb{R}^{n}\right) .
$$

In particular this implies that $\mathcal{H} \in L_{l o c}^{\infty}\left(\Omega, \mathbb{R}^{n-2}\right)$ and hence

$$
|\mathcal{H}|^{2} \in W_{l o c}^{1,2+\gamma}(\Omega)
$$

Now we can argue as in the proof of Corollary 3.7 in order to get

$$
u \in W_{l o c}^{3,2+\gamma}\left(\Omega, \mathbb{R}^{n-2}\right) .
$$

By the Sobolev embedding theorem this gives

$$
u \in C_{l o c}^{2, \beta}\left(\Omega, \mathbb{R}^{n-2}\right)
$$

for some $\beta>0$. The smoothness of solutions of equation (3.1) now follows from classical Schauder theory.

3.2. The functional $\mathcal{E}^{p}(f)$. Here we consider for $p>2$ weak solutions $u \in$ $W^{2, p}\left(\Omega, \mathbb{R}^{m}\right)$ of elliptic systems in two independent variables of the form

$$
\partial_{\alpha \beta}^{2}\left[a_{\alpha \beta}^{i}\left(\cdot, u, D u, D^{2} u\right)\right]+\partial_{\alpha}\left[b_{\alpha}^{i}\left(\cdot, u, D u, D^{2} u\right)\right]+c^{i}\left(\cdot, u, D u, D^{2} u\right)=0 .
$$

We assume that $a, b, c$ are $C^{1}$ functions satisfying the following ellipticity and growth conditions at all points $(x, z, p, q)$, for $V(x, z, p, q)=\left(1+|q|^{2}\right)^{1 / 2}$ and for constants $\lambda>0, C<\infty$ :

$$
\begin{gathered}
\frac{\partial a_{\alpha \beta}^{i}}{\partial q_{\lambda \mu}^{j}} \xi_{\alpha \beta}^{i} \xi_{\lambda \mu}^{j} \geq \lambda V^{p-2}|\xi|^{2} \\
\left|a_{q}\right| \leq C V^{p-2} \\
|a|+\left|a_{x}\right|+\left|a_{z}\right|+\left|a_{p}\right|+\left|b_{q}\right|+\left|c_{q}\right| \leq C V^{p-1} \\
|b|+\left|b_{x}\right|+\left|b_{z}\right|+\left|b_{p}\right|+|c|+\left|c_{x}\right|+\left|c_{z}\right|+\left|c_{p}\right| \leq C V^{p} .
\end{gathered}
$$

As noted in section 2, the graph function of a critical point for $\mathcal{E}^{p}$ satisfies a system of the required form, with suitable bounds (3.14) and (3.15). Therefore Theorem 
1.1 is a consequence of the following Proposition 3.8 and standard higher regularity theory, for which we refer to [16].

Proposition 3.8. Let $u \in W^{2, p}\left(\Omega, \mathbb{R}^{m}\right)$ be a weak solution of (3.13), where $p>2$ and $\Omega \subset \mathbb{R}^{2}$, and assume that (3.14) and (3.15) hold. Then $u$ belongs to $C_{\text {loc }}^{2, \alpha}\left(\Omega, \mathbb{R}^{m}\right)$ for some $\alpha>0$.

Remark 3.9. A related regularity result, for functionals where the integrand satisfies a more general (anisotropic) ellipticity condition but depends only on the second derivatives, was proved in 4]. A crucial ingredient both in [4 and in our paper is the Gehring type lemma from Bildhauer, Fuchs and Zhong [5].

3.2.1. Growth estimate. In a first step we show a growth estimate for the $L^{p}$-norm of the second derivatives of weak solutions of the system (3.13).

Lemma 3.10. Let $p>2$. There exist $r_{0}>0, \beta>0$ and $C>0$ such that if $u \in W^{2, p}\left(\Omega, \mathbb{R}^{m}\right)$ is a weak solution of the elliptic system (3.13) which satisfies (3.14) and (3.15), then we have for every $B_{2 r}(x) \subset \Omega$ with $r<r_{0}$ that

$$
\int_{B_{r}(x)} V^{p} \leq c\left(\frac{r}{r_{0}}\right)^{\beta}
$$

Proof. Let $r_{0}>0$. Since $u \in W^{2, p} \cap L^{\infty}\left(\Omega, \mathbb{R}^{m}\right)$ we get from the Sobolev embedding theorem that $u \in C^{1, \gamma}\left(\Omega, \mathbb{R}^{m}\right)$ for some $\gamma>0$. Now we choose $x_{0} \in \Omega$ and we let $0<2 r<\min \left\{2 r_{0}, \operatorname{dist}\left(x_{0}, \partial \Omega\right)\right\}$. Moreover we let $A_{r}=B_{2 r} \backslash B_{r}\left(x_{0}\right)$ and $\varphi \in C_{c}^{\infty}\left(B_{2 r}\left(x_{0}\right)\right)$ be a smooth cut-off function which satisfies

$$
0 \leq \varphi \leq 1, \quad \varphi=1 \text { in } B_{r}\left(x_{0}\right), \quad\left\|\nabla^{j} \varphi\right\|_{L^{\infty}} \leq c r^{-j} \forall j \in \mathbb{N} .
$$

Finally we define the linear function $l_{r}$ by

$$
l_{r}(x)=\frac{1}{\left|A_{r}\right|} \int_{A_{r}} u+\left(x-x_{0}\right) \cdot \frac{1}{\left|A_{r}\right|} \int_{A_{r}} D u .
$$

¿From this definition it easily follows that we have the estimates

$$
\begin{aligned}
\left\|u-l_{r}\right\|_{L^{\infty}\left(B_{2 r}\right)}+r\left\|D\left(u-l_{r}\right)\right\|_{L^{\infty}\left(B_{2 r}\right)} & \leq C r^{1+\gamma} \text { and } \\
\left\|u-l_{r}\right\|_{L^{p}\left(A_{r}\right)}+r\left\|D\left(u-l_{r}\right)\right\|_{L^{p}\left(A_{r}\right)} & \leq C r^{2}\left\|D^{2} u\right\|_{L^{p}\left(A_{r}\right)} .
\end{aligned}
$$

Now we choose $\varphi^{4}\left(u-l_{r}\right)$ as a test function in the weak form of the system (3.13) and we get

$$
\begin{aligned}
\left|\int_{\Omega} \varphi^{4} a_{\alpha \beta}^{i} \partial_{\alpha \beta}^{2} u^{i}\right| \leq & C \int_{\Omega} \varphi^{4}\left(|b|\left|D\left(u-l_{r}\right)\right|+|c|\left|u-l_{r}\right|\right) \\
& +C r^{-1} \int_{A_{r}} \varphi^{3}\left(|a|\left|D\left(u-l_{r}\right)\right|+|b|\left|u-l_{r}\right|\right) \\
& +C r^{-2} \int_{A_{r}} \varphi^{2}|a|\left|u-l_{r}\right| \\
= & I+I I+I I I .
\end{aligned}
$$


Using the ellipticity assumption and the bound $|a(x, z, p, 0)| \leq C$ (which follows from (3.15)) we estimate

$$
\begin{aligned}
q_{\alpha \beta}^{i} a_{\alpha \beta}^{i}(x, y, z, q) & =q_{\alpha \beta}^{i} a_{\alpha \beta}^{i}(x, y, z, 0)+q_{\alpha \beta}^{i} q_{\gamma \delta}^{j} \int_{0}^{1} a_{\alpha \beta, q_{\gamma \delta}^{j}}^{i}(x, y, z, t q) d t \\
& \geq q_{\alpha \beta}^{i} a_{\alpha \beta}^{i}(x, y, z, 0)+\lambda|q|^{2} \int_{0}^{1}\left(1+t^{2}|q|^{2}\right)^{\frac{p-2}{2}} d t \\
& \geq \tilde{\lambda} V^{p}-C
\end{aligned}
$$

where $\tilde{\lambda}>0$ is some number. Next we use (3.15), Hölders inequality and the estimates (3.18) and (3.19) to obtain

$$
\begin{aligned}
I & \leq C r^{\gamma} \int_{\Omega} \varphi^{4} V^{p}, \\
I I & \leq C r^{\gamma} \int_{A_{r}} V^{p}+C r^{-1}\|V\|_{L^{p}\left(A_{r}\right)}^{p-1}\left\|D\left(u-l_{r}\right)\right\|_{L^{p}\left(A_{r}\right)} \leq C \int_{A_{r}} V^{p} \text { and } \\
I I I & \leq C r^{-2}\left(\int_{A_{r}} V^{p}\right)^{\frac{p-1}{p}}\left\|u-l_{r}\right\|_{L^{p}\left(A_{r}\right)} \leq C \int_{A_{r}} V^{p} .
\end{aligned}
$$

Combining all these estimates and choosing $r_{0}$ small enough we conclude that there exists a constant $C_{1}>0$ such that

$$
\int_{B_{r}} V^{p} \leq C_{1} \int_{A_{r}} V^{p}+C r^{2}
$$

Adding $C_{1} \int_{B_{r}} V^{p}$ to both sides of this estimate we get

$$
\int_{B_{r}} V^{p} \leq \frac{C_{1}}{C_{1}+1} \int_{B_{2 r}} V^{p}+C r^{2} .
$$

The estimate (3.16) now follows from a standard iteration argument.

3.2.2. Difference quotient estimates. In a second step we use the difference quotient method to show that every weak solution $u \in W^{2, p} \cap L^{\infty}\left(\Omega, \mathbb{R}^{m}\right)$ of (3.13) is in $W^{3,2}\left(B_{\frac{r_{0}}{4}}\left(x_{0}\right), \mathbb{R}^{m}\right)$ and that moreover $U=V^{\frac{p}{2}} \in W^{1,2}\left(B_{\frac{r_{0}}{4}}\left(x_{0}\right)\right)$, where $x_{0} \in \Omega$ and $r_{0}$ is as in Lemma 3.10 (in the following we allow the constants to depend on $\left.r_{0}\right)$. We follow closely the methods developed in [16] and [19].

In the following we use the abbreviation $U(x)=\left(x, u(x), D u(x), D^{2} u(x)\right)$. Applying the difference quotient to the equation (3.13) and interchanging with the derivatives yields

$$
\partial_{\alpha \beta}^{2}\left[a_{\alpha \beta}^{i} \circ U\right]_{h}+\partial_{\alpha}\left[b_{\alpha}^{i} \circ U\right]_{h}+\left[c^{i} \circ U\right]_{h}=0 .
$$

We may use the fundamental theorem of Calculus to write

$$
\begin{aligned}
{[f \circ U]_{h}(x) } & =\frac{1}{h}\left(f\left(U\left(x+h e_{\nu}\right)\right)-f(U(x))\right) \\
& =\frac{1}{h} \int_{0}^{1} \frac{d}{d t} f\left((1-t) U(x)+t U\left(x+h e_{\nu}\right)\right) d t \\
& =\int_{0}^{1} D f\left((1-t) U(x)+t U\left(x+h e_{\nu}\right)\right) d t \cdot U_{h}(x) .
\end{aligned}
$$


Using the notation $f^{h}(x)=\int_{0}^{1} f\left((1-t) U(x)+t U\left(x+h e_{\nu}\right)\right) d t$ we thus get

$$
[f \circ U]_{h}=\left(\frac{\partial f}{\partial q_{\lambda \mu}^{j}}\right)^{h} \partial_{\lambda \mu}^{2} u_{h}^{j}+\left(\frac{\partial f}{\partial p_{\lambda}^{j}}\right)^{h} \partial_{\lambda} u_{h}^{j}+\left(\frac{\partial f}{\partial z^{j}}\right)^{h} u_{h}^{j}+\left(\frac{\partial f}{\partial x_{\nu}}\right)^{h},
$$

and the system (3.20) takes the form

$\partial_{\alpha \beta}^{2}\left[\tilde{a}_{\alpha \beta}^{i}\left(\cdot, u_{h}, D u_{h}, D^{2} u_{h}\right)\right]+\partial_{\alpha}\left[\tilde{b}_{\alpha}^{i}\left(\cdot, u_{h}, D u_{h}, D^{2} u_{h}\right)\right]+\tilde{c}^{i}\left(\cdot, u_{h}, D u_{h}, D^{2} u_{h}\right)=0$.

Here the coefficients are given as follows:

$$
\begin{aligned}
\tilde{a}_{\alpha \beta}^{i}(x, z, p, q)= & \left(\frac{\partial a_{\alpha \beta}^{i}}{\partial q_{\lambda \mu}^{j}}\right)^{h}(x) q_{\lambda \mu}^{j}+\left(\frac{\partial a_{\alpha \beta}^{i}}{\partial p_{\lambda}^{j}}\right)^{h}(x) p_{\lambda}^{j}+\left(\frac{\partial a_{\alpha \beta}^{i}}{\partial z^{j}}\right)^{h}(x) z^{j} \\
& +\left(\frac{\partial a_{\alpha \beta}^{i}}{\partial x_{\nu}}\right)^{h}(x), \\
\tilde{b}_{\alpha}^{i}(x, z, p, q)= & \left(\frac{\partial b_{\alpha}^{i}}{\partial q_{\lambda \mu}^{j}}\right)^{h}(x) q_{\lambda \mu}^{j}+\left(\frac{\partial b_{\alpha}^{i}}{\partial p_{\lambda}^{j}}\right)^{h}(x) p_{\lambda}^{j}+\left(\frac{\partial b_{\alpha}^{i}}{\partial z^{j}}\right)^{h}(x) z^{j}+\left(\frac{\partial b_{\alpha}^{i}}{\partial x_{\nu}}\right)^{h}(x), \\
\tilde{c}^{i}(x, z, p, q)= & \left(\frac{\partial c^{i}}{\partial q_{\lambda \mu}^{j}}\right)^{h}(x) q_{\lambda \mu}^{j}+\left(\frac{\partial c^{i}}{\partial p_{\lambda}^{j}}\right)^{h}(x) p_{\lambda}^{j}+\left(\frac{\partial c^{i}}{\partial z^{j}}\right)^{h}(x) z^{j}+\left(\frac{\partial c^{i}}{\partial x_{\nu}}\right)^{h}(x) .
\end{aligned}
$$

In order to state bounds for these coefficients, we introduce the abbreviation

$$
I_{s, h}(x)=\int_{0}^{1}\left(1+\left|(1-t) D^{2} u(x)+t D^{2} u(x+h)\right|^{2}\right)^{s / 2} d t .
$$

Using (3.14) and (3.15) we then obtain

$$
\begin{aligned}
|\tilde{a}(x, z, p, q)| & \leq C\left(I_{p-2, h}(x)|q|+I_{p-1, h}(x)(|p|+|z|+1)\right) \\
|\tilde{b}(x, z, p, q)|+|\tilde{c}(x, z, p, q)| & \leq C\left(I_{p-1, h}(x)|q|+I_{p, h}(x)(|p|+|z|+1)\right) .
\end{aligned}
$$

As above we define the linear function $l_{h, r}=: l_{h}$ by

$$
l_{h}(x)=\frac{1}{\left|A_{r}\right|} \int_{A_{r}} u_{h}+\left(x-x_{0}\right) \cdot \frac{1}{\left|A_{r}\right|} \int_{A_{r}} D u_{h} .
$$

Using again the test function $\varphi^{4}\left(u_{h}-l_{h}\right)$, we infer

$$
\begin{aligned}
\int \tilde{a}_{\alpha \beta}^{i} \partial_{\alpha \beta}^{2} u_{h}^{i} \varphi^{4} \leq & C \int\left(I_{p-1, h}\left|D^{2} u_{h}\right|+I_{p, h}\left(\left|D u_{h}\right|+\left|u_{h}\right|+1\right)\right) \\
& \cdot\left(\left|D\left(u_{h}-l_{h}\right)\right|+\left|u_{h}-l_{h}\right|\right) \varphi^{4} \\
& +\frac{C}{r} \int\left(I_{p-2, h}\left|D\left(u_{h}-l_{h}\right)\right|+I_{p-1, h}\left|u_{h}-l_{h}\right|\right)\left|D^{2} u_{h}\right| \varphi^{3} \\
& +\frac{C}{r} \int\left(I_{p-1, h}\left|D\left(u_{h}-l_{h}\right)\right|+I_{p, h}\left|u_{h}-l_{h}\right|\right) \\
& \cdot\left(\left|D u_{h}\right|+\left|u_{h}\right|+1\right) \varphi^{3} \\
& +\frac{C}{r^{2}} \int\left(I_{p-2, h}\left|D^{2} u_{h}\right|+I_{p-1, h}\left(\left|D u_{h}\right|+\left|u_{h}\right|+1\right)\right) \\
& \cdot\left|u_{h}-l_{h}\right| \varphi^{2} .
\end{aligned}
$$


On the other hand we have the ellipticity condition (using (3.14)

$$
\begin{aligned}
\tilde{a}_{\alpha \beta}^{i}(x, z, p, q) q_{\alpha \beta}^{i} & =\int_{0}^{1} \frac{\partial a_{\alpha \beta}^{i}}{\partial q_{\lambda \mu}^{j}}((1-t) U(x)+t U(x+h)) q_{\alpha \beta}^{i} q_{\lambda \mu}^{j} d t \\
& +\int_{0}^{1} \frac{\partial a_{\alpha \beta}^{i}}{\partial p_{\lambda}^{j}}((1-t) U(x)+t U(x+h)) p_{\lambda}^{j} q_{\alpha \beta}^{i} d t \\
& +\int_{0}^{1} \frac{\partial a_{\alpha \beta}^{i}}{\partial z^{j}}((1-t) U(x)+t U(x+h)) z^{j} q_{\alpha \beta}^{i} d t \\
& +\int_{0}^{1} \frac{\partial a_{\alpha \beta}^{i}}{\partial x_{\nu}}((1-t) U(x)+t U(x+h)) q_{\alpha \beta}^{i} d t \\
& \geq C \lambda I_{p-2, h}|q|^{2}-C I_{p-1, h}|q|(|p|+|z|+1) .
\end{aligned}
$$

Combining the two inequalities we arrive at

$$
\begin{aligned}
\int I_{p-2, h}\left|D^{2} u_{h}\right|^{2} \varphi^{4} \leq & C \int I_{p-1, h}\left|D^{2} u_{h}\right|\left(\left|D\left(u_{h}-l_{h}\right)\right| \varphi^{4}\right. \\
& \left.+\left|u_{h}-l_{h}\right|\left(\varphi^{4}+\frac{\varphi^{3}}{r}\right)+\left(\left|D u_{h}\right|+\left|u_{h}\right|+1\right) \varphi^{4}\right) \\
& +C \int I_{p-2, h}\left|D^{2} u_{h}\right|\left(\left|D\left(u_{h}-l_{h}\right)\right| \frac{\varphi^{3}}{r}+\left|u_{h}-l_{h}\right| \frac{\varphi^{2}}{r^{2}}\right) \\
& +C \int I_{p, h}\left(\left|D u_{h}\right|+\left|u_{h}\right|+1\right)\left(\left|D\left(u_{h}-l_{h}\right)\right| \varphi^{4}\right. \\
& \left.+\left|u_{h}-l_{h}\right|\left(\varphi^{4}+\frac{\varphi^{3}}{r}\right)\right) \\
& +C \int I_{p-1, h}\left(\left|D u_{h}\right|+\left|u_{h}\right|+1\right)\left(\left|D\left(u_{h}-l_{h}\right)\right| \frac{\varphi^{3}}{r}\right. \\
& \left.+\left|u_{h}-l_{h}\right| \frac{\varphi^{2}}{r^{2}}\right) .
\end{aligned}
$$

Using $I_{p-1, h} \leq I_{p-2, h}^{1 / 2} I_{p, h}^{1 / 2}$ and absorbing the second derivatives of $u_{h}$ yields

$$
\begin{aligned}
\int I_{p-2, h}\left|D^{2} u_{h}\right|^{2} \varphi^{4} \leq & C \int I_{p, h}\left(\left|D\left(u_{h}-l_{h}\right)\right|^{2}+\left|u_{h}-l_{h}\right|^{2}+\left|D u_{h}\right|^{2}\right. \\
& \left.+\left|u_{h}\right|^{2}+1\right) \varphi^{4}+C \int I_{p, h}\left|u_{h}-l_{h}\right|^{2} \frac{\varphi^{2}}{r^{2}} \\
& +C \int I_{p-2, h}\left(\left|D\left(u_{h}-l_{h}\right)\right|^{2} \frac{\chi_{A_{r}}}{r^{2}}+\left|u_{h}-l_{h}\right|^{2} \frac{\chi_{A_{r}}}{r^{4}}\right) \\
= & I+I I+I I I .
\end{aligned}
$$

Before continuing we need to recall the following Lemma which is essentially due to Morrey [16], Lemma 5.4.2. In the form stated here it can be found in [19].

Lemma 3.11. Let $r>0$ and let the function $q \geq 0$ be such that

$$
\int_{B_{s}(x) \cap B_{r}} q \leq c s^{\gamma}
$$


for all $B_{s}(x) \subset B_{r}$. Then for every $\varepsilon>0$ there exists $C_{\varepsilon}>0$ such that

$$
\int_{B_{r}} q|v|^{2} \leq \varepsilon r^{\gamma} \int_{B_{r}}|D v|^{2}+C_{\varepsilon} r^{\gamma-2} \int_{B_{r}}|v|^{2} .
$$

Next we use this Lemma in order to estimate the terms $I-I I I$ from above. By the definition of $I_{p, h}$ and Lemma 3.10 we get that

$$
\int_{B_{s}(x)} I_{p, h} \leq C s^{\beta}
$$

for all $B_{s}(x) \subset B_{2 r} \subset B_{r_{0}}$ and some $0<\beta<1$. Hence we can apply Lemma 3.11 and Hölder's respectively Poincaré's inequality to estimate

$$
I I \leq C r^{\beta-2}\left\|D\left(u_{h}-l_{h}\right)\right\|_{L^{2}\left(B_{2 r}\right)}^{2} .
$$

Using the same argument we get

$$
\begin{aligned}
I \leq & C r^{\beta} \int_{B_{2 r}} \varphi^{4}\left(\left|D^{2} u_{h}\right|^{2}+\left|D\left(u_{h}-l_{h}\right)\right|^{2}\right) \\
& +C r^{\beta-2} \int_{B_{2 r}}\left(\left|D u_{h}\right|^{2}+\left|D\left(u_{h}-l_{h}\right)\right|^{2}+\left|u_{h}\right|^{2}+\left|u_{h}-l_{h}\right|^{2}+I_{p, h}\right) .
\end{aligned}
$$

Inserting these two estimates into the above estimate for $\int I_{p-2, h}\left|D^{2} u_{h}\right|^{2} \varphi^{4}$ we conclude that

$$
\begin{aligned}
\int I_{p-2, h}\left|D^{2} u_{h}\right|^{2} \varphi^{4} \leq & C r^{\beta} \int_{B_{2 r}} \varphi^{4}\left(\left|D^{2} u_{h}\right|^{2}+\left|D\left(u_{h}-l_{h}\right)\right|^{2}\right)+C r^{\beta-2} \int_{B_{2 r}}\left(\left|D u_{h}\right|^{2}\right. \\
& \left.+\left|D\left(u_{h}-l_{h}\right)\right|^{2}+\left|u_{h}\right|^{2}+\left|u_{h}-l_{h}\right|^{2}+I_{p, h}\right) \\
& +C \int I_{p-2, h}\left(\left|D\left(u_{h}-l_{h}\right)\right|^{2} \frac{\chi_{A_{r}}}{r^{2}}+\left|u_{h}-l_{h}\right|^{2} \frac{\chi_{A_{r}}}{r^{4}}\right) .
\end{aligned}
$$

Next we use Hölder's and Poincaré's inequality to get

$$
\begin{aligned}
\int I_{p-2, h} & \left(\left|D\left(u_{h}-l_{h}\right)\right|^{2} \frac{\chi_{A_{r}}}{r^{2}}+\left|u_{h}-l_{h}\right|^{2} \frac{\chi_{A_{r}}}{r^{4}}\right) \\
\leq & \leq|| I_{p-2, h} \|_{L^{\frac{p}{p-2}}\left(B_{2 r}\right)}\left(r^{-4}\left\|u_{h}-l_{h}\right\|_{L^{p}\left(A_{r}\right)}^{2}+r^{-2}\left\|D\left(u_{h}-l_{h}\right)\right\|_{L^{p}\left(A_{r}\right)}\right) \\
\leq & C r^{-2}\left\|I_{p-2, h}\right\|_{L^{\frac{p}{p-2}}\left(B_{2 r}\right)}\left\|D\left(u_{h}-l_{h}\right)\right\|_{L^{p}\left(A_{r}\right)}^{2} .
\end{aligned}
$$

Since $u \in W^{2, p}(\Omega)$ we know from Theorem 3.6.8 in [16] that

$$
I_{s, h} \rightarrow V^{s} \quad \text { in } L^{\frac{p}{s}} \quad \forall 1 \leq s \leq p .
$$

We combine all the above estimates to get $\left(l_{h} \rightarrow f_{A_{r}} \partial_{\nu} u+\left(x-x_{0}\right) f_{A_{r}} \partial_{\nu} D u\right)$

$$
\int \varphi^{4} I_{p-2, h}\left|D^{2} u_{h}\right|^{2} \leq C\left(1+r^{-2}\right) \int_{B_{2 r}} V^{p}+C r^{\beta-2} \int_{B_{2 r}}\left|D^{2} u\right|^{2}+C r^{\beta} .
$$

In particular this estimate is true for $r=\frac{r_{0}}{4}$ and therefore we can let $h \rightarrow 0$ to conclude

$$
\int_{B_{\frac{r_{0}}{8}}} V^{p-2}\left|D^{3} u\right|^{2} \leq C\left(1+r_{0}^{-2}\right) \int_{B_{\frac{r_{0}}{4}}} V^{p}+c r_{0}^{\beta-2} \int_{\frac{r_{0}}{4}}\left|D^{2} u\right|^{2}+C r_{0}^{\beta} .
$$

Hence we have that $u \in W^{3,2}\left(B_{\frac{r_{0}}{8}}\right)$ and by the Sobolev embedding theorem this implies $u \in W_{\text {loc }}^{2, q}\left(B_{\frac{r_{0}}{8}}\right)$ for all $q<\infty$. Moreover the above estimate yields that 
$U \in W^{1,2}\left(B \frac{r_{0}}{8}\right)$. Altogether this shows that we can improve Lemma 3.10 to get the estimate

$$
\int_{B_{r}} V^{p} \leq c r^{2-\delta}
$$

for all $r \leq \frac{r_{0}}{16}$ and all $\delta>0$.

3.2.3. Higher regularity. In order to obtain the higher regularity for weak solutions of (3.13) we need to recall the following estimate from the previous subsection:

$$
\begin{aligned}
\int \varphi^{4} \tilde{a}_{\alpha \beta}^{i} \partial_{\alpha \beta}^{2} u_{h}^{i} \leq & C \int \varphi^{4}\left(I_{p-1, h}\left|D^{2} u_{h}\right|+I_{p, h}\left(\left|D u_{h}\right|+\left|u_{h}\right|+1\right)\right)\left(\left|D\left(u_{h}-l_{h}\right)\right|\right. \\
& \left.+\left|u_{h}-l_{h}\right|\right) \\
& +\frac{C}{r} \int \varphi^{3}\left(I_{p-2, h}\left|D\left(u_{h}-l_{h}\right)\right|+I_{p-1, h}\left|u_{h}-l_{h}\right|\right)\left|D^{2} u_{h}\right| \\
& +\frac{C}{r} \int \varphi^{3}\left(I_{p-1, h}\left|D\left(u_{h}-l_{h}\right)\right|+I_{p, h}\left|u_{h}-l_{h}\right|\right)\left(\left|D u_{h}\right|\right. \\
& \left.+\left|u_{h}\right|+1\right) \\
& +\frac{C}{r^{2}} \int \varphi^{2}\left(I_{p-2, h}\left|D^{2} u_{h}\right|+I_{p-1, h}\left(\left|D u_{h}\right|+\left|u_{h}\right|+1\right)\right)\left|u_{h}-l_{h}\right| \\
= & I+\ldots+I V .
\end{aligned}
$$

This time we choose $l_{h}$ such that

$$
\begin{aligned}
\int_{B_{2 r}}\left(u_{h}-l_{h}\right) & =0 \quad \text { and } \\
\int_{B_{2 r}}\left(D u_{h}-D l_{h}\right) & =0 .
\end{aligned}
$$

Because of (3.24) and the strong convergence $I_{s, h} \rightarrow V^{s}$ in $L^{\frac{p}{s}}$ we have for every $r \leq \frac{r_{0}}{16}$, every $h$ small enough and every $\delta>0$

$$
\int_{B_{r}} I_{p, h} \leq c r^{2-\delta}
$$

Now we estimate again each term seperately. We start with $I$. By Young's inequality we get

$$
\begin{aligned}
I \leq & \varepsilon \int \varphi^{4} I_{p-2, h}\left|D^{2} u_{h}\right|^{2}+C \int \varphi^{4} I_{p, h}\left(\left|D u_{h}\right|^{2}+\left|u_{h}\right|^{2}+1\right) \\
& +C \int \varphi^{4} I_{p, h}\left(\left|D\left(u_{h}-l_{h}\right)\right|^{2}+\left|u_{h}-l_{h}\right|^{2}\right)
\end{aligned}
$$

and we continue to estimate the last term with the help of Lemma 3.11, (3.25) and Poincaré's inequality by

$$
\begin{aligned}
\int \varphi^{4} I_{p, h}\left(\left|D\left(u_{h}-l_{h}\right)\right|^{2}+\left|u_{h}-l_{h}\right|^{2}\right) \leq & C r^{2-\delta} \int_{B_{2 r}}\left(\left|D^{2}\left(u_{h}-l_{h}\right)\right|^{2}+\left|D\left(u_{h}-l_{h}\right)\right|^{2}\right) \\
& +C r^{-\delta} \int_{B_{2 r}}\left(\left|D\left(u_{h}-l_{h}\right)\right|^{2}+\left|u_{h}-l_{h}\right|^{2}\right) \\
\leq & C r^{2-\delta} \int_{B_{2 r}}\left|D^{2} u_{h}\right|^{2} .
\end{aligned}
$$


Next we estimate

$$
\begin{aligned}
I I \leq & \varepsilon \int \varphi^{4} I_{p-2, h}\left|D^{2} u_{h}\right|^{2}+\frac{C}{r^{2}} \int_{B_{2 r}} I_{p, h}\left|u_{h}-l_{h}\right|^{2} \\
& +\frac{C}{r}\left(\int_{B_{2 r}} I_{p-2, h}^{\frac{4}{3}}\left|D^{2} u_{h}\right|^{\frac{4}{3}}\right)^{\frac{3}{4}}\left(\int_{B_{2 r}}\left|D\left(u_{h}-l_{h}\right)\right|^{4}\right)^{\frac{1}{4}} .
\end{aligned}
$$

The second term can be estimated as above to yield

$$
\frac{C}{r^{2}} \int_{B_{2 r}} I_{p, h}\left|u_{h}-l_{h}\right|^{2} \leq C r^{2-\delta} \int_{B_{2 r}}\left|D^{2} u_{h}\right|^{2}
$$

and for the third term we use the Sobolev-Poincaré inequality to get

$$
\frac{C}{r}\left(\int_{B_{2 r}} I_{p-2, h}^{\frac{4}{3}}\left|D^{2} u_{h}\right|^{\frac{4}{3}}\right)^{\frac{3}{4}}\left(\int_{B_{2 r}}\left|D\left(u_{h}-l_{h}\right)\right|^{4}\right)^{\frac{1}{4}} \leq \frac{C}{r}\left(\int_{B_{2 r}} I_{p-2, h}^{\frac{4}{3}}\left|D^{2} u_{h}\right|^{\frac{4}{3}}\right)^{\frac{3}{2}} .
$$

III can be estimated by

$$
\begin{aligned}
I I I \leq & \frac{\gamma}{r^{2}} \int_{B_{2 r}}\left(\left|D\left(u_{h}-l_{h}\right)\right|^{2}+I_{p, h}\left|u_{h}-l_{h}\right|^{2}\right) \\
& +C_{\gamma} \int_{B_{2 r}}\left(I_{2 p-2, h}+I_{p, h}\right)\left(\left|D u_{h}\right|^{2}+\left|u_{h}\right|^{2}+1\right) \\
\leq & C\left(\gamma+r^{2-\delta}\right) \int_{B_{2 r}}\left|D^{2} u_{h}\right|^{2}+C_{\gamma} \int_{B_{2 r}}\left(I_{2 p-2, h}+I_{p, h}\right)\left(\left|D u_{h}\right|^{2}+\left|u_{h}\right|^{2}+1\right) .
\end{aligned}
$$

Finally, using some of the estimates from above, the last term is estimated as follows

$$
\begin{aligned}
I V \leq & \frac{C}{r^{2}}\left(\int_{B_{2 r}} I_{p-2, h}^{\frac{4}{3}}\left|D^{2} u_{h}\right|^{\frac{4}{3}}\right)^{\frac{3}{4}}\left(\int_{B_{2 r}}\left|u_{h}-l_{h}\right|^{4}\right)^{\frac{1}{4}} \\
& +\frac{\gamma}{r^{4}} \int_{B_{2 r}}\left|u_{h}-l_{h}\right|^{2}+C_{\gamma} \int_{B_{2 r}} I_{2 p-2, h}\left(\left|D u_{h}\right|^{2}+\left|u_{h}\right|^{2}+1\right) \\
\leq & \frac{C}{r}\left(\int_{B_{r}} I_{p-2, h}^{\frac{4}{3}}\left|D^{2} u_{h}\right|^{\frac{4}{3}}\right)^{\frac{3}{2}}+C \gamma \int_{B_{2 r}}\left|D^{2} u_{h}\right|^{2} \\
& +C_{\gamma} \int_{B_{2 r}} I_{2 p-2, h}\left(\left|D u_{h}\right|^{2}+\left|u_{h}\right|^{2}+1\right) .
\end{aligned}
$$

We also note that we have the ellipticity estimate

$$
\int \varphi^{4} I_{p-2, h}\left|D^{2} u_{h}\right|^{2} \leq C \int \varphi^{4} \tilde{a}_{\alpha \beta}^{i} \partial_{\alpha \beta}^{2} u_{h}^{i}+C \int \varphi^{4} I_{p, h}\left(\left|D u_{h}\right|^{2}+\left|u_{h}\right|^{2}+1\right) .
$$

Combining all these estimates we get

$$
\begin{aligned}
\int_{B_{r}} I_{p-2, h}\left|D^{2} u_{h}\right|^{2} \leq & \frac{C}{r}\left(\int_{B_{2 r}} I_{p-2, h}^{\frac{4}{3}}\left|D^{2} u_{h}\right|^{\frac{4}{3}}\right)^{\frac{3}{2}}+C\left(\gamma+r^{2-\delta}\right) \int_{B_{2 r}}\left|D^{2} u_{h}\right|^{2} \\
& +C_{\gamma} \int_{B_{2 r}}\left(I_{2 p-2, h}+I_{p, h}\right)\left(\left|D u_{h}\right|^{2}+\left|u_{h}\right|^{2}+1\right) .
\end{aligned}
$$

Since $u \in W^{3,2}\left(B_{r}\right)$ and $U \in W^{1,2}\left(B_{r}\right)$ for $r \leq \frac{r_{0}}{16}$ we can let $h \rightarrow 0$ and get

$$
\begin{aligned}
\int_{B_{r}} V^{p-2}\left|D^{3} u\right|^{2} \leq & \frac{C}{r}\left(\int_{B_{2 r}} V^{\frac{4}{3}(p-2)}\left|D^{3} u\right|^{\frac{4}{3}}\right)^{\frac{3}{2}}+C\left(\gamma+r_{0}^{2-\delta}\right) \int_{B_{2 r}} V^{p-2}\left|D^{3} u\right|^{2} \\
& +C_{\gamma} \int_{B_{2 r}} V^{2 p}
\end{aligned}
$$


Defining $f=\left(V^{\frac{p-2}{2}}\left|D^{3} u\right|\right)^{4 / 3}, g=V^{\frac{2(p-2)}{3}}, h=V^{\frac{4 p}{3}}$ and $d=\frac{3}{2}$ we conclude the inequality

$$
\left(f_{B_{r}} f^{d}\right)^{\frac{1}{d}} \leq C f_{B_{2 r}} f g+C\left(\gamma+r_{0}^{2-\delta}\right)^{\frac{1}{d}}\left(f_{B_{2 r}} f^{d}\right)^{\frac{1}{d}}+C_{\gamma}\left(f_{B_{2 r}} h^{d}\right)^{\frac{1}{d}}
$$

for all balls $B_{r} \subset B_{\frac{r_{0}}{16}}$. Next we need the following Gehring type Lemma, which slightly generalizes Lemma 1.2 of Bildhauer, Fuchs and Zhong [5] (see also Theorem 1.1 in 9 ]).

Lemma 3.12. Let $d>1, \beta>0$ be two constants. There exists $\varepsilon_{0}>0$ such that for all all $\varepsilon<\varepsilon_{0}$ and all non-negative functions $f, g, h: \Omega \subset \mathbb{R}^{n} \rightarrow \mathbb{R}$ satisfying

$$
f, h \in L_{l o c}^{d}(\Omega), \quad e^{\beta g^{d}} \in L_{l o c}^{1}(\Omega)
$$

and (for some constant $C>0$ )

$$
\left(f_{B} f^{d}\right)^{\frac{1}{d}} \leq C f_{2 B} f g+\varepsilon\left(f_{2 B} f^{d}\right)^{\frac{1}{d}}+\left(f_{2 B} h^{d}\right)^{\frac{1}{d}}
$$

for all balls $B=B_{r}(x)$ with $B_{2 r}(x) \subset \subset \Omega$. Then there exists $c_{0}=c_{0}(n, d, C)>0$ such that if

$$
h^{d} \log ^{c_{0} \beta}(e+h) \in L_{l o c}^{1}(\Omega),
$$

then the same is true for $f$. Moreover, for all balls $B$ as above we have

$$
\begin{aligned}
f_{B} f^{d} \log ^{c_{0} \beta}\left(e+\frac{f}{\|f\|_{L^{d}(2 B)}}\right) \leq & c\left(f_{2 B} e^{\beta g^{d}}\right)\left(f_{2 B} f^{d}\right) \\
& +c f_{2 B} h^{d} \log ^{c_{0} \beta}\left(e+\frac{f}{\|f\|_{d, 2 B}}\right),
\end{aligned}
$$

where $c=c(n, d, \beta, C)>0$ and $\|f\|_{d, 2 B}=\left(f_{2 B} f^{d}\right)^{\frac{1}{d}}$.

Proof. The proof is very similar to the one of Lemma 1.2 in [5] and therefore we only comment on the differences.

We define $B_{0}=2 B$ and we assume without loss of generality that

$$
\int_{B_{0}} f^{d}=1
$$

Next we define the functions $d(x)=\operatorname{dist}\left(x, \mathbb{R}^{n} \backslash B_{0}\right)$ and

$$
\begin{aligned}
& \tilde{f}(x)=d(x)^{\frac{n}{d}} f, \\
& \tilde{h}(x)=d(x)^{\frac{n}{d}} h \quad \text { and } \\
& w(x)=\chi_{B_{0}}(x),
\end{aligned}
$$

where $\chi_{B_{0}}$ is the characteristic function of $B_{0}$. As in [5] it is now easy to see that because of (3.27) these new functions satisfy

$$
\begin{aligned}
\left(f_{B} \tilde{f}^{d}\right)^{\frac{1}{d}} \leq & C f_{2 B} \tilde{f} g+C \varepsilon\left(f_{2 B} \tilde{f}^{d}\right)^{\frac{1}{d}}+C\left(f_{2 B} \tilde{h}^{d}\right)^{\frac{1}{d}} \\
& +C\left(f_{2 B} w\right)^{\frac{1}{d}}
\end{aligned}
$$


and now this inequality is true for all balls $B \subset \mathbb{R}^{n}$. Hence, by taking the supremum over all radii, we get (here $M(f)$ denotes the maximal function of $f$ )

$$
M\left(\tilde{f}^{d}\right)^{\frac{1}{d}} \leq C M(\tilde{f} g)+C \varepsilon M\left(\tilde{f}^{d}\right)^{\frac{1}{d}}+C M\left(\tilde{h}^{d}\right)^{\frac{1}{d}}+C M(w)^{\frac{1}{d}} .
$$

For $\varepsilon_{0}$ small enough we therefore have

$$
M\left(\tilde{f}^{d}\right)^{\frac{1}{d}} \leq C M(\tilde{f} g)+C M\left(\tilde{h}^{d}\right)^{\frac{1}{d}}+C M(w)^{\frac{1}{d}}
$$

and with the help of this inequality we can copy the rest of the argument of the proof of Lemma 1.2 in [5] to finish the proof.

Now we want to apply this Lemma to our estimate (3.26). From the previous subsection we know that

$$
\begin{aligned}
& f^{d}=V^{p-2}\left|D^{3} u\right|^{2} \in L_{l o c}^{1}\left(B_{\frac{r_{0}}{16}}\right) \quad \text { and } \\
& h^{d}=V^{2 p} \in L_{l o c}^{1}\left(B_{\frac{r_{0}}{16}}\right) .
\end{aligned}
$$

Hence it remains to check that

$$
e^{\beta g^{d}}=e^{\beta V^{p-2}} \in L_{l o c}^{1}\left(B \frac{r_{0}}{16}\right)
$$

for some constant $\beta>0$. We actually claim that this is true for all $\beta>0$. In order to see this we note that by (3.23) we have that

$$
\int_{B \frac{r_{0}}{8}}\left|D\left(V^{\frac{p}{2}}\right)\right|^{2} \leq c_{1}\left(r_{0}\right)
$$

Next we let $\eta \in C_{c}^{\infty}\left(B_{\frac{r_{0}}{8}}\right)$ be a cut-off function such that $0 \leq \eta \leq 1, \eta(x) \equiv 1$ for all $x \in B_{\frac{r_{0}}{16}}$ and $\|D \eta\|_{L^{\infty}\left(B \frac{r_{0}}{8}\right)} \leq c r_{0}^{-1}$. Defining $v=\eta V^{\frac{p}{2}}$ we get that

$$
\int_{B_{\frac{r_{0}}{8}}}|D v|^{2} \leq c r_{0}^{-2} \int_{B_{\frac{r_{0}}{8}}} V^{p}+c \int_{B_{\frac{r_{0}}{8}}}\left|D V^{\frac{p}{2}}\right|^{2} \leq c_{2}\left(r_{0}\right) .
$$

Hence we see that $u=\frac{v}{\sqrt{c_{2}\left(r_{0}\right)}} \in H_{0}^{1}\left(B \frac{r_{0}}{8}\right)$ and

$$
\int_{\frac{r_{\frac{r_{0}}{8}}}{8}}|D u|^{2} \leq 1
$$

Therefore, by the Moser-Trudinger inequality (see 20]), there exist constants $\beta_{0}>0$ and $C=C\left(r_{0}\right)>0$ such that

$$
\int_{B_{\frac{r_{0}}{16}}} e^{\beta_{0} V^{p}} \leq \int_{B_{\frac{r_{0}}{8}}} e^{c_{2}\left(r_{0}\right) \beta_{0} u^{2}} \leq C .
$$

In particular this implies with the help of Young's inequality that for every $\beta>0$

$$
\int_{\frac{r_{0}}{16}} e^{\beta V^{p-2}} \leq c\left(\beta, \beta_{0}\right) \int_{\frac{r_{0}}{16}} e^{\beta_{0} V^{p}} \leq C\left(r_{0}, \beta, \beta_{0}\right) .
$$

Since we also have that

$$
h^{d} \log ^{\alpha}(e+h)=V^{2 p} \log ^{\alpha}\left(e+V^{\frac{4 p}{3}}\right) \in L_{l o c}^{1}\left(B_{\frac{r_{0}}{16}}\right)
$$

for every $\alpha>0$ we get from Lemma 3.12 that

$$
f^{d} \log ^{\alpha}(e+f) \in L_{l o c}^{1}\left(B_{\frac{r_{0}}{16}}\right)
$$


for every $\alpha>0$. Hence we obtain that

$$
\left|D^{3} u\right|^{2} \log ^{\alpha}\left(e+\left|D^{3} u\right|\right) \in L_{l o c}^{1}\left(B \frac{r_{0}}{16}\right)
$$

for every $\alpha>0$. In particular this is true for $\alpha>1$ and therefore we can apply Corollary 4.6 and Example 4.18(iv) of [8] (see also Example 5.3 in [11] for a different proof of this result) in order to conclude that

$$
u \in C^{2}\left(B \frac{r_{0}}{32}\right) .
$$

In particular this implies that

$$
\int_{B_{r}} V^{p} \leq c r^{2}
$$

for all $r \leq \frac{r_{0}}{32}$.

In order to show the Hölder continuity of $D^{2} u$ we go back to (3.22) and we estimate the last term with the help Lemma 3.11 and (3.30) by

$$
\begin{gathered}
\int I_{p-2, h}\left(\left|D\left(u_{h}-l_{h}\right)\right|^{2} \frac{\chi_{A_{r}}}{r^{2}}+\left|u_{h}-l_{h}\right|^{2} \frac{\chi_{A_{r}}}{r^{4}}\right) \\
\leq C \int_{A_{r}}\left|D^{2} u_{h}\right|^{2} .
\end{gathered}
$$

Inserting this estimate into (3.22), letting $h \rightarrow 0$ and using (3.30) we conclude for every $r \leq \frac{r_{0}}{32}$

$$
\int_{B_{r}}\left|D^{3} u\right|^{2} \leq C \int_{A_{r}}\left|D^{3} u\right|^{2}+C r^{\beta} .
$$

Remark 3.13. This estimate is sufficient for our purposes but by repeating all the estimates from subsection 3.2.2 and replacing every application of Lemma 3.10 by (3.30) one can actually improve this inequality in the sense that the term $r^{\beta}$ on the right hand side can be replaced by $r^{2}$.

The Hölder continuity of $D^{2} u$ now follows from (3.31) by another hole-filling argument. This finishes the proof of Proposition 3.8 .

\section{Compactness Results and existence of minimizers}

4.1. Compactness results. We start by quoting the fundamental compactness theorem of J. Langer (see also [6]).

Theorem 4.1. 14] Let $\Sigma$ be a closed surface and $p>2$. Assume that $f_{k} \in$ $W_{\mathrm{im}}^{2, p}\left(\Sigma, \mathbb{R}^{n}\right)$ is a sequence satisfying $0 \in f_{k}(\Sigma)$ for all $k \in \mathbb{N}$ and

$$
\mathcal{E}^{p}\left(f_{k}\right) \leq C \text {. }
$$

After replacing $f_{k}$ by $f_{k} \circ \varphi_{k}$ for suitable diffeomorphisms $\varphi_{k} \in C^{\infty}(\Sigma, \Sigma)$ and passing to a subsequence, the $f_{k}$ converge weakly in $W^{2, p}\left(\Sigma, \mathbb{R}^{n}\right)$ to an $f \in W_{\mathrm{im}}^{2, p}\left(\Sigma, \mathbb{R}^{n}\right)$. In particular, the convergence is in $C^{1, \beta}\left(\Sigma, \mathbb{R}^{n}\right)$ for any $\beta<1-\frac{2}{p}$, and

$$
\mathcal{E}^{p}(f) \leq \liminf _{k \rightarrow \infty} \mathcal{E}^{p}\left(f_{k}\right) .
$$


In this section we prove Theorem 1.2, which replaces the $\mathcal{E}^{p}$ bound in Langer's theorem by a bound only for $\mathcal{W}^{p}$, under the additional assumption that the Willmore energy is bounded below $8 \pi$. Before entering the proof we include two remarks about the statement.

Remark 4.2. One can allow sequences $f_{k}: \Sigma_{k} \rightarrow \mathbb{R}^{n}$ in Theorem 1.2, where $\Sigma_{k}$ are arbitrary closed oriented surfaces. In fact, a bound on the genus follows from the condition $\liminf _{k \rightarrow \infty} \mathcal{W}\left(f_{k}\right)<8 \pi$ by a result of Kuwert, Li 8 Schätzle [13].

Remark 4.3. Connecting two round spheres by a shrinking catenoid neck yields a sequence of smoothly embedded surfaces with bounded $\mathcal{W}^{p}$-energy and Willmore energy less than $8 \pi$. As the convergence is not in $C^{1}$, this shows that the assumption on the Willmore energy in Theorem 1.2 cannot be weakened. Similar constructions are also possible for higher genus, see Kühnel \& Pinkall [12] and Simon [19].

To prove Theorem 1.2 we need the following area ratio bounds, which are immediate consequences of Simon's monotonicity identity [19].

Lemma 4.4. Let $f: \Sigma \rightarrow \mathbb{R}^{n}$ be an embedded closed surface. Then

$$
\sigma^{-2}\left|\Sigma \cap B_{\sigma}\right| \leq C \mathcal{W}(f) \quad \text { for all } \sigma>0 \text {. }
$$

Moreover for any $p>2$ we have the estimate

$$
\sigma^{-2}\left|\Sigma \cap B_{\sigma}\right| \leq \frac{1}{4} \mathcal{W}(f)+C \sigma^{\frac{p-2}{p}} \quad \text { for all } \sigma>0
$$

where the constant $C$ depends on $\mathcal{W}^{p}(f)$.

Proof. By equation (1.2) in [19, we have for $0<\sigma<\infty$ the inequality

$$
\begin{aligned}
\sigma^{-2}\left|\Sigma \cap B_{\sigma}\right| \leq & \rho^{-2}\left|\Sigma \cap B_{\rho}\right|+\frac{1}{4} \mathcal{W}(f) \\
& +\frac{1}{2} \int_{\Sigma \cap B_{\rho}} \rho^{-2}\langle x, H\rangle d \mu-\frac{1}{2} \int_{\Sigma \cap B_{\sigma}} \sigma^{-2}\langle x, H\rangle d \mu .
\end{aligned}
$$

Letting $\rho \rightarrow \infty$ we conclude for every $\sigma>0$

$$
\sigma^{-2}\left|\Sigma \cap B_{\sigma}\right| \leq \frac{1}{4} \mathcal{W}(f)+\frac{1}{2 \sigma} \int_{\Sigma \cap B_{\sigma}}|H| d \mu .
$$

The estimates now follow from an application of Hölder's inequality.

As second ingredient, we need the following lemma yielding an $L^{p}$ estimate for the prescribed mean curvature system.

Lemma 4.5. Let $u \in W^{2, p}\left(B_{\varrho}, \mathbb{R}^{k}\right)$, where $B_{\varrho}=\left\{x \in \mathbb{R}^{2}:|x|<\varrho\right\}$ and $0<\varrho<$ $\infty, p \in(1, \infty)$, be a solution of the system

$$
a_{i j}^{\alpha \beta} \partial_{\alpha \beta}^{2} u^{i}=\varphi_{j} \quad \text { for } j=1, \ldots, k .
$$

There is an $\varepsilon_{0}=\varepsilon_{0}(p)>0$ such that if

$$
\left|a_{i j}^{\alpha \beta}(x)-\delta^{\alpha \beta} \delta_{i j}\right| \leq \varepsilon_{0} \quad \text { for all } x \in B_{\varrho},
$$

then for some $C=C(p)<\infty$ we have the estimate

$$
\left\|D^{2} u\right\|_{L^{p}\left(B_{\frac{\rho}{2}}\right)} \leq C\left(\|\varphi\|_{L^{p}\left(B_{\varrho}\right)}+\varrho^{-1}\|D u\|_{L^{p}\left(B_{\varrho}\right)}\right) .
$$


Proof. We may assume that $\varrho=1$ and that $u$ has mean value zero on $B_{1}$. For $\eta \in C_{0}^{\infty}\left(B_{1}\right)$ satisfying $\eta=1$ on $B_{\frac{1}{2}}$ and $\eta=0$ in $B_{1} \backslash B_{\frac{3}{4}}$, we calculate

$$
a_{i j}^{\alpha \beta} \partial_{\alpha \beta}^{2}\left(\eta u^{i}\right)=\eta \varphi^{j}+a_{i j}^{\alpha \beta}\left(\partial_{\alpha \beta}^{2} \eta\right) u^{i}+a_{i j}^{\alpha \beta}\left(\partial_{\alpha} \eta \partial_{\beta} u^{i}+\partial_{\beta} \eta \partial_{\alpha} u^{i}\right) .
$$

Hence we have

$$
\begin{aligned}
\Delta\left(\eta u^{j}\right)= & \left(\delta^{\alpha \beta} \delta_{i j}-a_{i j}^{\alpha \beta}\right) \partial_{\alpha \beta}^{2}\left(\eta u^{i}\right)+\eta \varphi^{j} \\
& +a_{i j}^{\alpha \beta}\left(\partial_{\alpha \beta}^{2} \eta\right) u^{i}+a_{i j}^{\alpha \beta}\left(\partial_{\alpha} \eta \partial_{\beta} u^{i}+\partial_{\beta} \eta \partial_{\alpha} u^{i}\right) .
\end{aligned}
$$

¿From standard $L^{p}$-estimates and the Poincaré inequality we obtain

$$
\left\|D^{2}(\eta u)\right\|_{L^{p}\left(B_{1}\right)} \leq C \varepsilon_{0}\left\|D^{2}(\eta u)\right\|_{L^{p}\left(B_{1}\right)}+C\left(\|\varphi\|_{L^{p}\left(B_{1}\right)}+\|D u\|_{L^{p}\left(B_{1}\right)}\right),
$$

for a constant $C=C(p)<\infty$. This shows the desired result.

Proof of Theorem 1.2, Let $f_{k}: \Sigma \rightarrow \mathbb{R}^{n}$ be a sequence as in the theorem. For each $q \in \Sigma$, we let $r_{k}(q)>0$ be the maximal radius on which $f_{k}$ is represented as a graph over the tangent plane at $q$. We denote by $u_{k, q}: B_{r_{k}(q)} \rightarrow \mathbb{R}^{n-2}$ the corresponding graph function, obtained by choosing a suitable rigid motion. In particular

$$
u_{k, q}(0)=0 \quad \text { and } \quad D u_{k, q}(0)=0 .
$$

For $\varepsilon>0$ we define $r_{k}(q, \varepsilon)=\sup \left\{r \in\left(0, r_{k}(q)\right]:\left\|D u_{k, q}\right\|_{C^{0}\left(B_{r}\right)}<\varepsilon\right\}$ and

$$
r_{k}=\inf _{q \in \Sigma} r_{k}(q, \varepsilon) \text {. }
$$

By compactness, the infimum is attained at some point $q_{k} \in \Sigma$ and we have $r_{k}>0$. We will show by contradiction that

$$
\liminf _{k \rightarrow \infty} r_{k}>0 .
$$

Assuming that $r_{k} \rightarrow 0$ we rescale by putting

$$
\tilde{f}_{k}: \Sigma \rightarrow \mathbb{R}^{n}, \tilde{f}_{k}(p)=\frac{1}{r_{k}}\left(f_{k}(p)-f_{k}\left(q_{k}\right)\right) .
$$

Clearly, the $\tilde{f}_{k}$ have local graph representations

$$
\tilde{u}_{k, q}: B_{r_{k}(q) / r_{k}} \rightarrow \mathbb{R}^{n-2}, \tilde{u}_{k, q}(x)=\frac{1}{r_{k}} u_{k, q}\left(r_{k} x\right),
$$

where $\tilde{u}_{k, q}(0)=0$ and $D \tilde{u}_{k, q}(0)=0$, and

$$
\left\|\tilde{u}_{k, q}\right\|_{C^{0}\left(B_{1}\right)}+\left\|D \tilde{u}_{k, q}\right\|_{C^{0}\left(B_{1}\right)} \leq C \varepsilon .
$$

¿From the bound $\mathcal{W}^{p}\left(f_{k}\right) \leq C$ we further infer that

$$
\int_{\Sigma}\left|H_{\tilde{f}_{k}}\right|^{p} d \mu_{\tilde{f}_{k}}=r_{k}^{p-2} \int_{\Sigma}\left|H_{f_{k}}\right|^{p} d \mu_{f_{k}} \leq C r_{k}^{p-2} \rightarrow 0 .
$$

The prescribed mean curvature system (3.2) for the $u_{k}$ fulfills the assumption of Lemma 4.5 if $\varepsilon=\varepsilon(p)>0$ is sufficiently small. Therefore we get the $L^{p}$ estimate

$$
\left\|D^{2} \tilde{u}_{k, q}\right\|_{L^{p}\left(B_{\frac{1}{2}}\right)} \leq C\left(\left\|H_{\tilde{f}_{k}}\right\|_{L^{p}\left(B_{1}\right)}+\left\|D \tilde{u}_{k, q}\right\|_{L^{p}\left(B_{1}\right)}\right) \leq C .
$$

Moreover the monotonicity formula (4.3) yields for $B_{R}=B_{R}(0) \subset \mathbb{R}^{n}$

$$
\mu_{\tilde{f}_{k}}\left(B_{R}\right) \leq C R^{2} \quad \text { for any } R \in(0, \infty) \text {. }
$$


We now apply a localized version of Langer's theorem from [6] to obtain a proper immersion $f_{0}: \Sigma_{0} \rightarrow \mathbb{R}^{n}$, such that the $\tilde{f}_{k}$ converge to $f_{0}$ locally in $C^{1, \beta}$ up to diffeomorphisms. Weak lower semicontinuity of $\mathcal{W}^{p}$ implies

$$
H_{f_{0}}=0 .
$$

The Gauß-Bonnet theorem yields

$$
\int_{\Sigma}\left|A_{f_{k}}\right|^{2} d \mu_{f_{k}}=4 \mathcal{W}\left(f_{k}\right)-4 \pi \chi(\Sigma) \leq C,
$$

and thus we get further

$$
\int_{\Sigma_{0}}\left|A_{f_{0}}\right|^{2} d \mu_{f_{0}} \leq \liminf _{k \rightarrow \infty} \int_{\Sigma}\left|A_{\tilde{f}_{k}}\right|^{2} d \mu_{\tilde{f_{k}}} \leq C .
$$

Summarizing, we have that $f_{0}: \Sigma_{0} \rightarrow \mathbb{R}^{n}$ is a properly immersed minimal surface with finite total curvature. By results of Chern \& Osserman, see [7], the immersion $f_{0}$ admits a conformal reparametrisation on a compact surface with finitely many punctures, corresponding to the ends. Moreover, each end has a well-defined tangent plane and multiplicity. Now the monotonicity formula from (4.5) implies

$$
\frac{\mu_{\tilde{f}_{k}}\left(B_{R}\right)}{\pi R^{2}}=\frac{\mu_{f_{k}}\left(B_{r_{k} R}\right)}{\pi\left(R r_{k}\right)^{2}} \leq \frac{1}{4} \mathcal{W}\left(f_{k}\right)+C\left(R r_{k}\right)^{\frac{p-2}{p}} \quad \text { for all } R>0
$$

Letting $k \rightarrow \infty$ and then $R \rightarrow \infty$ we conclude that

$$
\limsup _{R \rightarrow \infty} \frac{\mu_{f_{0}}\left(B_{R}\right)}{\pi R^{2}}<2
$$

This means that $f_{0}$ has just one simple end, and is in fact a plane. We now argue that the Gauß map converges to a constant locally uniformly on $\Sigma_{0}=\mathbb{R}^{2}$, contradicting the definition of $r_{k}$. More precisely, from the compactness theorem in [6] we know that $\tilde{f}_{k} \circ \phi_{k} \rightarrow f_{0}$ locally in $C^{1}$ and moreover

$$
\left\|\tilde{f}_{k} \circ \phi_{k}-f_{0}\right\|_{C^{0}\left(U_{k}\right)} \rightarrow 0
$$

where the $U_{k} \subset \mathbb{R}^{2}$ are open sets with $U_{1} \subset U_{2} \subset \ldots$ and $\mathbb{R}^{2}=\bigcup_{k=1}^{\infty} U_{k}$, such that $\phi_{k}: U_{k} \rightarrow \tilde{f}_{k}^{-1}\left(B_{k}(0)\right)$ is diffeomorphic. Now $\tilde{f}_{k}\left(q_{k}\right)=0$ by construction, therefore there exists a $p_{k} \in U_{k}$ with $\phi_{k}\left(p_{k}\right)=q_{k}$. In particular we have

$$
f_{0}\left(p_{k}\right)=f_{0}\left(p_{k}\right)-\left(\tilde{f}_{k} \circ \phi_{k}\right)\left(p_{k}\right) \rightarrow 0 .
$$

Since $f_{0}$ is proper, we get $p_{k} \rightarrow p \in \mathbb{R}^{2}$ after passing to a subsequence. Now by the indirect assumption, there exist $x_{k} \in B_{1}(0)$ such that for all $k$

$$
\left|D \tilde{u}_{k, q_{k}}\left(x_{k}\right)-D \tilde{u}_{k, q_{k}}(0)\right| \geq \frac{\varepsilon}{2}>0 .
$$

Denote the corresponding point by $q_{k}^{\prime} \in \Sigma$. Then $\left|\tilde{f}_{k}\left(q_{k}^{\prime}\right)\right| \leq C$, and hence there are points $p_{k}^{\prime} \in \mathbb{R}^{2}$ with $\phi_{k}\left(p_{k}^{\prime}\right)=q_{k}^{\prime}$. This implies

$$
\left|f\left(p_{k}^{\prime}\right)\right| \leq\left|f\left(p_{k}^{\prime}\right)-\left(\tilde{f}_{k} \circ \phi_{k}\right)\left(p_{k}^{\prime}\right)\right|+\left|\tilde{f}_{k}\left(q_{k}^{\prime}\right)\right| \leq C .
$$

Using again that $f_{0}$ is proper, we conclude after passing to a further subsequence that $p_{k}^{\prime} \rightarrow p^{\prime} \in \mathbb{R}^{2}$. But now $T_{p} f=\lim _{k \rightarrow \infty} T_{p_{k}}\left(\tilde{f}_{k} \circ \phi_{k}\right)=\lim _{k \rightarrow \infty} T_{q_{k}} \tilde{f}_{k}$, and analogously $T_{p^{\prime}} f=\lim _{k \rightarrow \infty} T_{q_{k}^{\prime}} \tilde{f}_{k}$. From the indirect assumption, we obtain $T_{p} f \neq T_{p^{\prime}} f$, contradicting the fact that $f$ parametrizes a plane. 
Given (4.6) we may finally use Lemma 4.5 with $\varrho:=\inf _{k \in \mathbb{N}} r_{k}>0$ to get

$$
\int_{B_{\frac{\rho}{2}}}\left|D^{2} u_{k, q}\right|^{p} \leq C \quad \text { for all } q \in \Sigma, k \in \mathbb{N} .
$$

The global mass bound and a standard covering argument then imply that

$$
\mathcal{E}^{p}\left(f_{k}\right) \leq C \quad \text { for all } k \in \mathbb{N} .
$$

The desired conclusion now follows from Theorem 4.1

4.2. Existence of minimizers. Combining Theorem 4.1 with our regularity from Theorem 1.1 we immediately get

Theorem 4.6. For a closed surface $\Sigma$ and $p>2$, denote by $\alpha_{\Sigma}^{n}(p)$ the infimum of the energy $\mathcal{E}^{p}$ among all smooth immersions from $\Sigma$ into $\mathbb{R}^{n}$. Then $\alpha_{\Sigma}^{n}(p)$ is attained by a smooth immersion $f: \Sigma \rightarrow \mathbb{R}^{n}$.

Proof. Using mollification it is easy to see that

$$
\alpha_{\Sigma}^{n}(p)=\inf \left\{\mathcal{E}^{p}(f): f \in W_{\mathrm{im}}^{2, p}\left(\Sigma, \mathbb{R}^{n}\right)\right\} .
$$

Thus the limiting map $f \in W_{\mathrm{im}}^{2, p}\left(\Sigma, \mathbb{R}^{n}\right)$ of a minimizing sequence obtained from Theorem 4.1 is a critical point of $\mathcal{E}^{p}$, and hence smooth after composing with a diffeomorphism by Theorem 1.1 .

For any fixed immersion $f: \Sigma \rightarrow \mathbb{R}^{n}$ we have

$$
\lim _{q \rightarrow p} \alpha_{\Sigma}^{n}(q) \leq \lim _{q \rightarrow p} \mathcal{E}^{q}(f)=\mathcal{E}^{p}(f) .
$$

Taking the infimum with respect to $f$ shows that the function $\alpha_{\Sigma}^{n}:[2, \infty) \rightarrow \mathbb{R}$ is upper semicontinuous. In particular, the function is continuous from the right since it is nondecreasing. For $\lambda>0$ and $f: \Sigma \rightarrow \mathbb{R}^{n}$ fixed we also note

$$
\alpha_{\Sigma}^{n}(2) \leq \mathcal{E}^{2}(\lambda f)=\frac{\lambda^{2}}{4} \mu_{g}(\Sigma)+\frac{1}{4} \int_{\Sigma}|A|^{2} d \mu_{g} \leq \frac{\lambda^{2}}{4} \mu_{g}(\Sigma)+\mathcal{E}^{2}(f) .
$$

Letting $\lambda \searrow 0$ and taking the infimum with respect to $f$ shows

$$
\alpha_{\Sigma}^{n}(2)=\inf _{f: \Sigma \rightarrow \mathbb{R}^{n}} \frac{1}{4} \int_{\Sigma}|A|^{2} d \mu_{g}
$$

Recall that by the Gauß equation and the Gauß-Bonnet theorem

$$
\mathcal{W}(f)=\frac{1}{4} \int_{\Sigma}|A|^{2} d \mu_{g}+\pi \chi(\Sigma) .
$$

The infimum of the Willmore energy among immersions of $\Sigma$ into $\mathbb{R}^{n}$ satisfies $\beta_{\Sigma}^{n}<8 \pi$ [2]. Thus for $p>2$ close to 2, we conclude for a minimizer $f$ of $\mathcal{E}^{p}$ that

$$
\mathcal{W}(f) \leq \mathcal{E}^{p}(f)+\pi \chi(\Sigma)=\alpha_{\Sigma}^{n}(p)+\pi \chi(\Sigma)<8 \pi .
$$

In particular, these minimizers are embedded by the Li-Yau inequality [15].

Next we define the number $\beta_{\Sigma}^{n}(p)$ as the infimum of the energy $\mathcal{W}^{p}$ among all smooth immersions from $\Sigma$ into $\mathbb{R}^{n}$. Repeating the previous discussion with $\beta_{\Sigma}^{n}$ 
instead of $\alpha_{\Sigma}^{n}$ we conclude that for every sequence of immersions $f_{k}: \Sigma \rightarrow \mathbb{R}^{n}$ with $\mathcal{W}^{p}\left(f_{k}\right) \rightarrow \beta_{\Sigma}^{n}$ and $p-2$ small enough we have

$$
\mathcal{W}\left(f_{k}\right) \leq \mathcal{W}^{p}\left(f_{k}\right) \rightarrow \beta_{\Sigma}^{n}(p)<8 \pi
$$

Combing this estimate with Theorem 1.2 . Theorem 3.1 and arguing as in the proof of Theorem 4.6 we get

Theorem 4.7. There exists $2<p_{0}<\infty$ such that for every closed surface $\Sigma$ and every $2<p<p_{0}$ the number $\beta_{\Sigma}^{n}(p)$ is attained by a smooth immersion $f: \Sigma \rightarrow \mathbb{R}^{n}$.

The numbers $\alpha_{\Sigma}^{n}(p)$ and $\beta_{\Sigma}^{n}(p)$ depend only on the topological type of $\Sigma$. This can be refined by minimizing in regular homotopy classes of immersions $f: \Sigma \rightarrow \mathbb{R}^{n}$. Theorem 4.6 and Theorem 4.7 extend without any difficulties.

\section{Palais-Smale condition}

Here we show that for $p>2$ the functionals $\mathcal{E}^{p}$ resp. $\mathcal{W}^{p}$ satisfy the Palais-Smale condition resp. a modified Palais-Smale condition, up to the action of diffeomorphisms on $\Sigma$. For $f \in W_{\text {im }}^{2, p}\left(\Sigma, \mathbb{R}^{n}\right)$ and any $V \in W^{2, p}\left(\Sigma, \mathbb{R}^{n}\right)$ we define the norm

$$
\|V\|_{W_{f}^{2, p}(\Sigma)}=\left(\int_{\Sigma}\left(|\nabla(D V)|_{g}^{p}+|D V|_{g}^{p}+|V|^{p}\right) d \mu_{g}\right)^{\frac{1}{p}},
$$

where $g \in W^{1, p}\left(T^{0,2} \Sigma\right)$ is the metric induced by $f$ and $\nabla$ denotes its Levi-Cività connection, with Christoffel symbols locally in $L^{p}$. In particular, the norm is welldefined. Now put

$$
\left\|D \mathcal{E}^{p}(f)\right\|_{f}=\sup \left\{D \mathcal{E}^{p}(f) V: V \in W^{2, p}\left(\Sigma, \mathbb{R}^{n}\right),\|V\|_{W_{f}^{2, p}(\Sigma)} \leq 1\right\},
$$

resp.

$$
\left\|D \mathcal{W}^{p}(f)\right\|_{f}=\sup \left\{D \mathcal{W}^{p}(f) V: V \in W^{2, p}\left(\Sigma, \mathbb{R}^{n}\right),\|V\|_{W_{f}^{2, p}(\Sigma)} \leq 1\right\},
$$

For any diffeomorphism $\varphi \in W^{2, p}(\Sigma, \Sigma)$ we have that $(f \circ \varphi)^{*} g_{\text {euc }}=\varphi^{*}\left(f^{*} g_{\text {euc }}\right)$, which implies $\|V \circ \varphi\|_{f \circ \varphi}=\|V\|_{f}$ and therefore

$$
\left\|D \mathcal{E}^{p}(f \circ \varphi)\right\|_{f \circ \varphi}=\left\|D \mathcal{E}^{p}(f)\right\|_{f},
$$

resp.

$$
\left\|D \mathcal{W}^{p}(f \circ \varphi)\right\|_{f \circ \varphi}=\left\|D \mathcal{W}^{p}(f)\right\|_{f},
$$

Now we can formulate the main results of this section.

Theorem 5.1. Let $f_{k} \in W_{\mathrm{im}}^{2, p}\left(\Sigma, \mathbb{R}^{n}\right), p>2$, be a sequence satisfying

$$
\mathcal{E}^{p}\left(f_{k}\right) \leq C \quad \text { and } \quad\left\|D \mathcal{E}^{p}\left(f_{k}\right)\right\|_{f_{k}} \rightarrow 0 .
$$

Then, after choosing a subsequence and passing to $f_{k} \circ \varphi_{k}$ for suitable diffeomorphisms $\varphi_{k} \in C^{\infty}(\Sigma, \Sigma)$, the $f_{k}$ converge strongly in $W^{2, p}\left(\Sigma, \mathbb{R}^{n}\right)$ to some $f \in W_{\mathrm{im}}^{2, p}\left(\Sigma, \mathbb{R}^{n}\right)$, and $f$ is a smooth critical point of $\mathcal{E}^{p}$.

Theorem 5.2. Let $f_{k} \in W_{\mathrm{im}}^{2, p}\left(\Sigma, \mathbb{R}^{n}\right), \delta>0, p>2$, be a sequence satisfying

$$
\mathcal{W}^{p}\left(f_{k}\right) \leq C, \quad \mathcal{W}\left(f_{k}\right) \leq 8 \pi-\delta \quad \text { and } \quad\left\|D \mathcal{W}^{p}\left(f_{k}\right)\right\|_{f_{k}} \rightarrow 0 .
$$

Then, after choosing a subsequence and passing to $f_{k} \circ \varphi_{k}$ for suitable diffeomorphisms $\varphi_{k} \in C^{\infty}(\Sigma, \Sigma)$, the $f_{k}$ converge strongly in $W^{2, p}\left(\Sigma, \mathbb{R}^{n}\right)$ to some $f \in W_{\mathrm{im}}^{2, p}\left(\Sigma, \mathbb{R}^{n}\right)$, and $f$ is a smooth critical point of $\mathcal{W}^{p}$. 
Since the arguments for the two results are very similar (thanks to Theorem 4.1 and Theorem 1.2) we only present the proof of Theorem 5.1 .

Proof. Langer's compactness theorem [14 yields that after passing to a subsequence $f_{k} \circ \varphi_{k} \rightarrow f$ in the $C^{1}$ topology and weakly in $W^{2, p}\left(\Sigma, \mathbb{R}^{n}\right)$, where $f \in W_{\text {im }}^{2, p}\left(\Sigma, \mathbb{R}^{n}\right)$ and $\varphi_{k} \in C^{\infty}(\Sigma, \Sigma)$ are diffeomorphisms. It remains to see that the convergence is strong in $W^{2, p}\left(\Sigma, \mathbb{R}^{n}\right)$, for which it suffices to consider the local convergence of the graph representations over a disk $B_{r} \subset \mathbb{R}^{2}$. Namely, then the assumption implies that $f$ is a critical point of $\mathcal{E}^{p}$ and is hence smooth by Theorem 1.1, after composing with a further diffeomorphism.

Let $u_{k}, u \in W^{2, p}\left(B_{r}, \mathbb{R}^{n-2}\right)$ be the graph functions for $f_{k}$ and $f$, respectively. Then $u_{k} \rightarrow u$ in $C^{1}\left(B_{r}\right)$, weakly in $W^{2, p}\left(B_{r}\right)$, and we can assume

$$
\left\|D u_{k}\right\|_{C^{0}\left(B_{r}\right)} \leq L \leq 1 \quad \text { and }\left\|u_{k}\right\|_{W^{2, p}\left(B_{r}\right)}^{p} \leq C \mathcal{E}^{p}\left(f_{k}\right) \leq C .
$$

We let $\psi_{k}=\eta\left(u_{k}-u\right)$ where $\chi_{B_{r / 2}} \leq \eta \leq \chi_{B_{r}}$ is a cut-off function. Clearly

$$
\left\|\psi_{k}\right\|_{C^{1}\left(B_{r}\right)} \rightarrow 0 \quad \text { and } \quad\left\|\psi_{k}\right\|_{W^{2, p}\left(B_{r}\right)} \leq C .
$$

Next we recall from (2.2) the Fréchet derivative, in a graph representation:

$$
D \mathcal{E}^{p}\left(f_{k}\right)\left(0, \psi_{k}\right)=\int_{B_{r}}\left(a_{i}^{\alpha \beta}\left(D u_{k}, D^{2} u_{k}\right) \partial_{\alpha \beta}^{2} \psi_{k}^{i}+b_{i}^{\alpha}\left(D u_{k}, D^{2} u_{k}\right) \partial_{\alpha} \psi_{k}^{i}\right),
$$

where

$$
\begin{aligned}
a_{i}^{\alpha \beta}\left(D u_{k}, D^{2} u_{k}\right)= & \frac{p}{4}\left(1+\left|A_{k}\right|^{2}\right)^{\frac{p-2}{2}} B_{i j}^{\alpha \beta, \gamma \lambda}\left(D u_{k}\right) \partial_{\gamma \lambda}^{2} u_{k}^{j} \sqrt{\operatorname{det} g_{k}}, \\
b_{i}^{\alpha}\left(D u_{k}, D^{2} u_{k}\right)= & \frac{p}{8}\left(1+\left|A_{k}\right|^{2}\right)^{\frac{p-2}{2}} \frac{\partial B_{j m}^{\gamma \lambda, \mu \nu}}{\partial p_{\alpha}^{i}}\left(D u_{k}\right) \partial_{\gamma \lambda}^{2} u_{k}^{j} \partial_{\mu \nu} u_{k}^{m} \sqrt{\operatorname{det} g_{k}} \\
& +\frac{1}{4}\left(1+\left|A_{k}\right|^{2}\right)^{\frac{p}{2}} \frac{\partial \sqrt{\operatorname{det} g_{k}}}{\partial p_{\alpha}^{i}}\left(D u_{k}\right) .
\end{aligned}
$$

Here $\left(g_{k}\right)_{\alpha \beta}=\delta_{\alpha \beta}+\left\langle\partial_{\alpha} u_{k}, \partial_{\beta} u_{k}\right\rangle$ and $B_{i j}^{\alpha \beta, \gamma \lambda}\left(D u_{k}\right)=g_{k}^{\alpha \gamma} g_{k}^{\beta \lambda}\left(\delta_{i j}-g_{k}^{\mu \nu} \partial_{\mu} u_{k}^{i} \partial_{\nu} u_{k}^{j}\right)$. We see easily that

$$
\begin{aligned}
a_{i}^{\alpha \beta}\left(D u_{k}, D^{2} u_{k}\right) & \leq C\left(1+\left|D^{2} u_{k}\right|^{2}\right)^{\frac{p-1}{2}}, \\
b_{i}^{\alpha}\left(D u_{k}, D^{2} u_{k}\right) & \leq C L\left(1+\left|D^{2} u_{k}\right|^{2}\right)^{\frac{p}{2}},
\end{aligned}
$$

and obtain for $k \rightarrow \infty$

$$
\begin{aligned}
\int_{B_{r}} a_{i}^{\alpha \beta}\left(D u_{k}, D^{2} u_{k}\right)\left(\partial_{\alpha \beta}^{2} \psi_{k}^{i}-\eta \partial_{\alpha \beta}^{2}\left(u_{k}^{i}-u^{i}\right)\right) & \rightarrow 0, \\
\int_{B_{r}} b_{i}^{\alpha}\left(D u_{k}, D^{2} u_{k}\right) \partial_{\alpha} \psi_{k}^{i} & \rightarrow 0 .
\end{aligned}
$$

Now using (5.3) and (5.4) we get $\left\|\left(0, \psi_{k}\right)\right\|_{W_{f_{k}}^{2, p}(\Sigma)} \leq C\left\|\psi_{k}\right\|_{W^{2, p}} \leq C$, and hence

$$
D \mathcal{E}^{p}\left(f_{k}\right)\left(0, \psi_{k}\right) \rightarrow 0 \quad \text { as } k \rightarrow \infty,
$$

using the assumption of the theorem and (5.1). Combining with (5.6) and (5.7), and noting that $a_{i}^{\alpha \beta}\left(D u, D^{2} u\right) \in L^{p}\left(B_{r}, \mathbb{R}^{n-2}\right)^{\prime}$, we conclude

$$
\int_{B_{r}} \eta\left(a_{i}^{\alpha \beta}\left(D u_{k}, D^{2} u_{k}\right)-a_{i}^{\alpha \beta}\left(D u, D^{2} u\right)\right) \partial_{\alpha \beta}^{2}\left(u_{k}^{i}-u^{i}\right) \rightarrow 0 \quad \text { as } k \rightarrow \infty .
$$


But since $u_{k} \rightarrow u$ in $C^{1}\left(B_{r}, \mathbb{R}^{n-2}\right)$ we also have that

$$
\int_{B_{r}} \eta\left(a_{i}^{\alpha \beta}\left(D u_{k}, D^{2} u_{k}\right)-a_{i}^{\alpha \beta}\left(D u, D^{2} u_{k}\right)\right) \partial_{\alpha \beta}^{2}\left(u_{k}^{i}-u^{i}\right) \rightarrow 0,
$$

and by adding the last two equations we get

$$
\int_{B_{r}} \eta\left(a_{i}^{\alpha \beta}\left(D u, D^{2} u_{k}\right)-a_{i}^{\alpha \beta}\left(D u, D^{2} u\right)\right) \partial_{\alpha \beta}^{2}\left(u_{k}^{i}-u^{i}\right) \rightarrow 0 \quad \text { as } k \rightarrow \infty .
$$

Finally we use the ellipticity, see (3.14), to estimate

$$
\begin{aligned}
& \int_{B_{r}} \eta\left(a_{i}^{\alpha \beta}\left(D u, D^{2} u_{k}\right)-a_{i}^{\alpha \beta}\left(D u, D^{2} u\right)\right) \partial_{\alpha \beta}^{2}\left(u_{k}^{i}-u^{i}\right) \\
= & \int_{B_{r}} \eta \int_{0}^{1} \frac{\partial a_{i}^{\alpha \beta}}{\partial q_{\lambda \mu}^{j}}\left(D u, D^{2} u+t D^{2}\left(u_{k}-u\right)\right) \partial_{\lambda \mu}^{2}\left(u_{k}^{j}-u^{j}\right) \partial_{\alpha \beta}^{2}\left(u_{k}^{i}-u^{i}\right) d t \\
\geq & \lambda \int_{B_{r}} \eta \int_{0}^{1}\left(1+\left|D^{2} u+t D^{2}\left(u_{k}-u\right)\right|^{2}\right)^{\frac{p-2}{2}}\left|D^{2}\left(u_{k}-u\right)\right|^{2} d t \\
\geq & c \lambda \int_{B_{r / 2}}\left|D^{2}\left(u_{k}-u\right)\right|^{p} .
\end{aligned}
$$

In the last step we used the elementary Lemma 19.27 from [17. Altogether we have proved local and hence global convergence in $W^{2, p}$.

\section{REFERENCES}

[1] L. Ambrosio and S. Masnou. A direct variational approach to a problem arising in image reconstruction. Interfaces Free Bound., 5:63-81, 2003.

[2] M. Bauer and E. Kuwert. Existence of minimizing Willmore surfaces of prescribed genus. Int. Math. Res. Not., 10:553-576, 2003.

[3] G. Bellettini, G. Dal Maso and M. Paolini. Semicontinuity and relaxation properties of a curvature depending functional in 2D. Ann. Scuola Norm. Sup. Pisa Cl. Sci., 20:247-297, 1993.

[4] M. Bildhauer and M. Fuchs. Higher order variational problems on two-dimensional domains. Ann. Acad. Sci. Fenn. Math., 31:349-362, 2006.

[5] M. Bildhauer, M. Fuchs and X. Zhong. A lemma on the higher integrabilty of functions with applications to the regularity theory of two-dimensional generalized Newtonian fluids. Manuscripta Math., 116:135-156, 2005.

[6] P. Breuning. Immersions with local Lipschitz representation. PhD thesis Freiburg, 2011

[7] S.S. Chern and R. Osserman. Complete minimal surfaces in Euclidean $n$-space. J. Analyse Math., 19:15-34, 1967.

[8] D. Edmunds, P. Gurka and B. Opic. On embeddings of logarithmic Bessel potential spaces. J. Funct. Anal., 146:116-150, 1997.

[9] D. Faraco, P. Koskela and X. Zhong. Mappings of finite distortion: the degree of regularity. Adv. Math., 190:300-318, 2005.

[10] D. Gilbarg, N. Trudinger. Elliptic partial differential equations of second order. Die Grundlehren der mathematischen Wissenschaften, Band 224, Springer Verlag New York, New York, 1998.

[11] J. Kauhanen, P. Koskela and J. Maly. On functions with derivatives in a Lorentz space. Manuscripta Math., 100:87-101, 1999.

[12] W. Kühnel and U. Pinkall. On total mean curvatures Quart. J. Math. Oxford Ser. (2), 37:437-447, 1986.

[13] E. Kuwert, Y. Li and R. Schätzle. The large genus limit of the infimum of the Willmore energy. Amer. J. Math., 132:37-52, 2010.

[14] J. Langer. A compactness theorem for surfaces with $L^{p}$-bounded second fundamental form. Math. Ann., 270:223-234, 1985. 
[15] P. Li and S.T. Yau. A new conformal invariant and its applications to the Willmore conjecture and the first eigenvalue on compact surfaces. Invent. Math., 69:269-291, 1982.

[16] C.B. Morrey. Multiple integrals in the calculus of variations. Die Grundlehren der mathematischen Wissenschaften, Band 130, Springer Verlag New York, New York, 1966.

[17] R. Palais. Foundations of global non-linear analysis. W.A. Benjamin, Inc., New YorkAmsterdam, 1968.

[18] J. Sacks and K. Uhlenbeck. The existence of minimal immersions of 2-spheres. Ann. of Math., 113:1-24, 1981.

[19] L. Simon. Existence of surfaces minimizing the Willmore functional. Comm. Anal. Geom., 1:281-326, 1993.

[20] N. Trudinger. On imbeddings into Orlicz spaces and some applications. J. Math. Mech., 17:473-483, 1967.

(E. Kuwert) Mathematisches Institut, Universität Freiburg, Eckerstrasse 1, 79104 Freiburg, Germany

E-mail address: ernst.kuwert@math.uni-freiburg.de

(T. Lamm) Institut Für Mathematik, Goethe-Universität Frankfurt, Robert-Mayer-Str. 10, 60054 Frankfurt, Germany

E-mail address: lamm@math.uni-frankfurt.de

(Y. Li) Department of Mathematical Sciences, Tsinghua University, Beijing 100084, P.R.CHINA

E-mail address: yxli@math.tsinghua.edu.cn 